\title{
NONLINEAR EVOLUTION OF TIDALLY DISTORTED ACCRETION DISKS: TWO-DIMENSIONAL SIMULATIONS
}

\author{
DONGSU RYU ${ }^{1}$ AND JEREMY GOODMAN ${ }^{2}$ \\ Princeton University Observatory, Peyton Hall, Princeton, NJ 08544 \\ Received 1993 February 16; accepted 1993 August 16
}

\begin{abstract}
According to a previously published linear analysis, the tidal distortion of accretion disks in binary star systems produces a local hydrodynamic instability to $m=1$ internal waves, which may have arbitrarily small wavelengths in the absence of viscosity. The instability is three-dimensional and approximately incompressible. To explore the nonlinear outcome of this instability, we develop a shearing-sheet approximation on scales comparable to the disk thickness. The large-scale azimuthal variation of the disk is represented by varying the local metric with time (local orbital phase). The hydrodynamic equations can then be posed two-dimensionally on local meridional planes. We solve these equations with a second-order gasdynamical code based on the Total-Variation-Diminishing scheme.

Our simulations confirm the predicted linear growth rate. The modes saturate chaotically at velocities scaling as the product of the linear growth rate and the wavelength. If the wavelength is small compared with the disk thickness, the modes remain nearly incompressible even when nonlinear. The two-dimensional power spectrum of velocities after saturation is roughly isotropic and extends over a broad range of scales in an approximately power-law fashion.

We measure the heating rate associated with the nonlinear dissipation of the modes. The dissipation implies a secular torque on the disk and a return of angular momentum to the secondary star via the tidal potential. The estimated torque is somewhat larger than the tidal torque produced by maximal disk viscosity $(\alpha \sim 1)$. At least in these two-dimensional simulations, however, there is no significant angular momentum flux within the disk.

Subject headings: accretion, accretion disks — hydrodynamics — instabilities
\end{abstract}

\section{INTRODUCTION}

This is the second in a series of papers devoted to a local, purely hydrodynamical instability of accretion disks that will be referred to here as the "elliptical instability." The series itself is only part of a larger campaign currently being waged by many investigators to understand the nature and causes of turbulence and angular momentum transport in accretion disks.

The linear theory of the elliptical instability as it applies to disks was developed by Goodman (1993, hereafter Paper I) and further studied by Lubow, Pringle, \& Kerswell (1993, hereafter LPK). In the present paper we explore the nonlinear development and saturation of the instability using a quasi-axisymmetric approximation. Within this approximation, our numerical simulations allow us to confirm the earlier analytic work in the linear regime and, at saturation, to measure the local rate of exchange of angular momentum between the disk and the secondary (mass-donating) star. We hope to report on fully three-dimensional simulations in a subsequent paper.

We begin with a summary of the main ideas and results of Paper I.

It was shown that an inviscid and nonmagnetic circular disk possesses an almost continuous spectrum of axisymmetric inertial oscillations, the restoring force for which is due to the conservation of angular momentum. These modes are generalized epicyclic motions, to which they reduce in the special case that the perturbed velocities are entirely horizontal (that is, perpendicular to the rotation axis $z$ ). In a fluid disk, epicyclic oscillations can occur in planes inclined to the horizontal by an arbitrary angle $\theta$. The component of the Coriolis force normal to such a plane is balanced by the perturbed pressure gradient.

In the limit that the wavelength of such a mode is very small compared with the disk thickness, the inertial oscillations are almost incompressible. The WKB wavenumber, $\boldsymbol{k}$, must therefore be perpendicular to the perturbed velocities, hence normal to the plane of motion. The axisymmetric dispersion relation is

$$
\omega^{2}=\kappa^{2} \cos ^{2} \theta \equiv \kappa^{2} \frac{k_{z}^{2}}{k_{z}^{2}+k_{r}^{2}},
$$

where $\boldsymbol{k}=k_{r} e_{r}+k_{z} e_{z}$, and $\kappa$ is the epicyclic frequency. If the disk is vertically stratified, the term $N^{2} \sin ^{2} \theta$ must be added to the right-hand side of equation (1), where $N^{2}$ is the local Brunt-Väisälä frequency. As in Paper I, we take $N^{2}=0$, which should always be true near the disk midplane. ${ }^{3}$

\footnotetext{
${ }_{1}^{1}$ Present address: Chungnam National University, Department of Astronomy and Space Science, Daejeon, 305-764 South Korea.

${ }^{2}$ David and Lucille Packard Foundation Fellow.

${ }^{3}$ LPK consider an isothermal vertical structure, for which $N^{2}>0$, but they simplify the tidal distortion itself. These differences in approach make it difficult to compare their work with Paper I quantitatively, although both papers find an instability.
} 
The dispersion relation (1) can be generalized to mildly nonaxisymmetric modes of azimuthal symmetry $m \ll r k$ (the most interesting case for our purposes is $m=1$ ):

$$
\omega^{\prime 2} \equiv[\omega-m \Omega(r)]^{2}=\kappa^{2} \cos ^{2} \theta,
$$

where $\Omega(r)$ is the local orbital frequency of the disk. The quantity $\omega^{\prime}$ is simply the frequency of the mode measured in a frame corotating with the disk.

It is important to note that the magnitude of the wavenumber $\boldsymbol{k}$ does not figure in the dispersion relations (1) and (2)-only its orientation does. Thus inertial oscillations of a given frequency can exist over a broad range of scales. Compressibility changes the dispersion relation for wavelengths $\lambda \gtrsim H$, and viscosity rapidly damps oscillations at wavelengths $\lambda \lesssim(v / \kappa)^{1 / 2}$.

If there is a secondary star orbiting outside the disk, it will tidally distort the disk in a predominantly $m_{\text {tide }}=2$ pattern. If the mass ratio $\left(M_{1} / M_{2}\right)$ of the primary to the secondary star is large, the distortion can be regarded as a long-wavelength, coherent, forced epicyclic motion at frequency $\omega_{\text {tide }}=m_{\text {tide }} \Omega_{b}$, where $\Omega_{b} \equiv\left[G\left(M_{1}+M_{2}\right) / a^{3}\right]^{1 / 2}$ is the orbital frequency of the binary, and $a$ is its semimajor axis. As viewed by an observer co-orbiting with the disk, the forcing frequency is

$$
\omega_{\text {tide }}^{\prime}=\omega_{\text {tide }}-m_{\text {tide }} \Omega(r)=m_{\text {tide }}\left[\Omega_{b}-\Omega(r)\right] .
$$

Unless the disk extends to the inner Lindblad radius, we have by definition that $\left|\omega_{\text {tide }}^{\prime}\right|>\kappa(r)$, so the tidal forcing is not directly resonant with the modified epicyclic motions described by equation (2). But we should also consider the possibility of a parametric resonance.

The conditions for (exact) parametric resonance are

$$
\left|\omega_{\text {mode }}^{\prime}\right|=\frac{1}{2}\left|\omega_{\text {tide }}^{\prime}\right|, \quad\left|m_{\text {mode }}\right|=\frac{1}{2}\left|m_{\text {tide }}\right| \text {. }
$$

(Higher order resonances can exist with $\frac{1}{2}$ replaced by $1 / n$ in eq. [4], provided that $m_{\text {mode }}$ is an integer, but are very much weaker.) The first of conditions (4) is directly analogous to the condition for parametric resonance in harmonic oscillators (cf. Landau $\&$ Lifshitz 1967). We can rewrite it as

$$
\omega_{\text {mode }} \pm \frac{1}{2} \omega_{\text {tide }}=\left(m_{\text {mode }} \pm \frac{1}{2} m_{\text {tide }}\right) \Omega(r),
$$

with correlated signs. Since the frequencies in the nonrotating frame are independent of $r$, the coefficient of $\Omega(r)$ in equation (5) must vanish. Thus, the second of conditions (4) follows from the first and the assumption of a coherent global mode. Therefore, $m_{\text {mode }}= \pm 1$ for the dominant $m_{\text {tide }}=2$ component of the tide. If $\kappa(r) \approx \Omega(r)$ (an approximately Keplerian disk) and $m_{\text {tide }}=2$, then equation (3) implies that $\left|\omega_{\text {tide }}^{\prime}\right| \leq 2 \kappa(r)$; this means that the first of conditions (4) is satisfied for a physically permissible choice of $\cos \theta$ in the dispersion relation (2). The pattern speed of a resonant mode in the nonrotating frame, $\Omega_{p} \equiv \operatorname{Re}\left(\omega_{\text {mode }} / m_{\text {mode }}\right)$, is simply $\Omega_{b}$. In summary, therefore, an $m=2$ tidal distortion is parametrically resonant with selected $|m|=1$ inertial oscillations at every radius in a Keplerian disk.

To establish that this parametric resonance leads to a finite growth rate, it is not enough to point to a coincidence of frequencies: one must calculate the coupling between the modes and the tidally distorted background. This was done in Paper I, where it was shown that the local growth rate at small radii $(r \ll a)$ is

$$
\operatorname{Im}\left(\omega_{\text {mode }}\right) \approx \frac{15}{4} \mu \frac{\Omega_{b}^{2}}{\Omega(r)}
$$

where $\mu \equiv M_{2} /\left(M_{1}+M_{2}\right)$. The growth rate is somewhat larger than predicted by formula (6) when $r / a$ is not small.

These instabilities occur on small scales because $k_{z}$ is quantized in units of order $\pi H^{-1}$, where $H$ is the half-thickness of the disk, and $k_{z} \neq 0$ unless $\theta=\pi / 2$. With present-day computer resources, it is probably impossible to simulate an entire thin disk with sufficient resolution to see these modes. We are therefore forced to make a local approximation on scales $\lesssim H$ that somehow incorporates the large-scale tidal effects. It is shown in $\S 2$ that this is indeed possible. Furthermore, we can reduce the problem to two spatial dimensions, because both the tidal distortion and the destabilized linear modes are almost axisymmetric when viewed on scales of order $\mathrm{H}$; of course, by so doing we limit the form of the fluid motions in the nonlinear regime, and therefore the conclusions that we draw about the nonlinear behavior must be regarded as provisional until confirmed by three-dimensional work.

The local hydrodynamical equations are derived and presented in $\S 2$. In $\S 3$ we describe the numerical method that we have developed to solve these equations. Tests of our code are also described, including a three-dimensional simulation of an elliptical vortex in a box. Results from two-dimensional simulations of the disk instability are presented in $\S 4$, and $\S 5$ sums up.

\section{THE SHEARING SHEET FOR A TIDALLY DISTORTED DISK}

In this section we reduce the fluid equations from a global, curvilinear coordinate system to a local, pseudo-Cartesian "shearingsheet" system. We also derive an expression for the momentum flux.

\subsection{Derivation of the Local Fluid Equations}

We assume that there exists a solution for the disk that is stationary in a reference frame on the primary and corotating with binary orbit. The streamlines of the stationary solution are closed, and we adopt a coordinate system based upon them: that is, two of the three spatial coordinates are constant on streamlines, and the time derivative of the third coordinate is constant following the fluid. The fluid equations for a general flow (not just the stationary one) are written out in this system. We then expand the equations in a coordinate neighborhood of a fiducial point that travels along a streamline of the stationary flow. The small parameter of this expansion is the ratio of the size of the neighborhood to the streamline radius, $\bar{r}_{0}$. We assume that the ratio of the perturbed velocities $(\delta v)$ to the orbital velocity $\left(\bar{r}_{0} \Omega\right)$ is of the same order as this small parameter. Note, however, that our local approximation 
is not a linearization. We are not assuming, for example, that $\delta v \cdot \nabla \delta v \ll \partial_{t} \delta v$, because although $\delta v$ is small, its spatial derivatives may not be.

In the reference frame described above, the inviscid Euler equation is

$$
\partial_{t} v+v \cdot \nabla v+2 \Omega_{b} \times v+\nabla U+\nabla w=0 .
$$

If the primary lies at $r=r_{1}=0$ and the secondary at $r=r_{2}$, then the effective potential is

$$
U(\boldsymbol{r}) \equiv-\frac{G M_{1}}{r}-\frac{G M_{2}}{\left|r-r_{2}\right|}-\frac{1}{2}\left|\Omega_{b} \times r\right|^{2}+\frac{G M_{2}}{r_{2}}\left(1+\frac{r_{2} \cdot r}{r_{2}^{2}}\right) .
$$

The final terms on the right occur because we work in a frame that is "freely falling" with the primary; these terms remove the monopole and dipole parts of the gravitational field of the secondary.

We have temporarily assumed an isentropic disk (that is, $p \propto \rho^{\gamma}$, where $\rho$ and $p$ are the density and pressure of the fluid), and we have introduced the enthalpy,

$$
w \equiv \frac{\gamma p}{(\gamma-1) \rho}
$$

It follows that $\nabla w=\rho^{-1} \nabla p$.

Equation (7) can be written in covariant form as

$$
\partial_{t} v^{i}+v^{j} v_{; j}^{i}+M_{j}^{i} v^{j}+g^{i j}(U+w)_{, j} .
$$

Our tensor notation follows Weinberg (1972): $\left\{x^{i} \mid i \in 1,2,3\right)$ are arbitrary spatial coordinates, subscript commas denote partial derivatives with respect to these coordinates, and subscript semicolons denote covariant derivatives. That is,

$$
v_{; j}^{i} \equiv v_{, j}^{i}+\Gamma_{j k}^{i} v^{k}
$$

where the affine connections $\Gamma_{j k}^{j}$ transform between coordinate systems in such a way as to make $v_{; j}^{i}$ transform as a tensor. In cylindrical coordinates $(r, \phi, z)$, the nonzero components of the affine connection are

$$
\Gamma_{r \phi}^{\phi}=\Gamma_{\phi r}^{\phi}=\frac{1}{r}, \quad \Gamma_{\phi \phi}^{r}=-r .
$$

The quantity $g^{i j}$ is the inverse of the metric tensor, which is diagonal in cylindrical coordinates:

$$
g^{r r}=g^{z z}=1, \quad g^{\phi \phi}=\frac{1}{r^{2}} .
$$

The tensor $M^{i}{ }_{j}$ describes the Coriolis force. In a cylindrical system whose $z$-axis is aligned with $\boldsymbol{\Omega}_{b}$,

$$
M^{i}{ }_{j}=2 \Omega_{b}\left(r^{-1} \delta_{\phi}^{i} \delta_{j}^{r}-r \delta_{r}^{i} \delta_{j}^{\phi}\right) .
$$

To first order in the tidal distortion, streamline coordinates can be defined by writing

$$
r=\bar{r}+\xi^{r}, \quad z=\bar{z}+\xi^{z}, \quad \phi=\bar{\phi}+\xi^{\phi},
$$

and requiring that the average along streamlines of $\xi^{r}, \xi^{z}$, and $\xi^{\phi}$ should vanish. To first order in the tide, it does not matter whether the streamline average is taken with respect to time, arc length, or $\phi$. Each streamline is labeled by a constant value of $\bar{r}$ and $\bar{z}$. Thus the coordinate velocities of the stationary (but noncircular) disk are

$$
\bar{v}^{\bar{r}}=\bar{v}^{\bar{z}}=0, \quad \overline{v^{\Phi}}=\Omega(\bar{r})-\Omega_{b},
$$

where $\Omega(\bar{r})$ is the mean angular velocity along the streamline in a nonrotating frame. The displacements $\left\{\xi^{i}\right\}$ are functions of the $\left\{\bar{x}^{i}\right\}$. We assume that $|\xi| \ll r$, but we do not assume $|\xi| \lesssim h$, where $h$ is a scale $\lesssim H$, the disk thickness. In what follows, we discard all terms beyond the first order in $\xi$.

It can be shown (cf. Weinberg 1972) that the metric and affine connection in the barred system are related in first order to those of cylindrical coordinates by

$$
\begin{aligned}
\bar{g}^{i j}(\bar{x}) & =\frac{\partial \bar{x}^{i}}{\partial x^{k}} \frac{\partial \bar{x}^{j}}{\partial x^{l}} g^{k l}(\bar{x}+\xi)=g^{i j}(\bar{x})-g^{k j} \xi_{, k}^{i}-g^{i l} \xi_{, l}^{j}+\xi^{n} g^{i j}, \\
\bar{\Gamma}_{j k}^{i}(\bar{x}) & =\Gamma_{j k}^{i}(\bar{x})+\xi_{: j: k}^{i} .
\end{aligned}
$$

The metric and connections on the right-hand sides of equations (17a) and (17b) have the functional forms of the corresponding quantities in cylindrical coordinates, but their arguments have been replaced by the barred variables; that is, $g^{i j}(\bar{x}) \equiv \operatorname{diag}\left(1,1, \bar{r}^{-2}\right)$ and, for example, $\Gamma_{\phi \phi}^{r}(\bar{x})=-\bar{r}$. In equation $(17 \mathrm{~b}), \xi$ is differentiated as a vector field, and we have used colons rather than semicolons to indicate that the covariant derivatives of $\xi$ are to be evaluated using $\Gamma(\bar{x})$ rather $\operatorname{than} \bar{\Gamma}(\bar{x})$.

With this machinery, we can divide $(v \cdot \nabla v)$ in the barred system into parts of zeroth and first order in $\xi$ :

$$
\bar{v}^{j} \bar{v}_{; j}^{i}=\bar{v}^{j} \bar{v}_{: j}^{i}+\xi_{: j: k}^{i} \bar{v}^{j} \bar{v}^{k}
$$

We can use equation (17a) to divide the term $\bar{g}^{i j}(U+w)_{, j}$ similarly. The Coriolis tensor (eq. [15]) expands to

$$
\bar{M}_{j}^{i}=\frac{\partial \bar{x}^{i}}{\partial x^{k}} \frac{\partial x^{l}}{\partial \bar{x}^{j}} M_{l}^{k}(\bar{x}+\xi)=2 \Omega_{b}\left[\left(\bar{r}^{-1} \delta_{\bar{\phi}}^{i} \delta_{j}^{\bar{r}}-\bar{r} \delta_{\bar{r}}^{i} \delta_{j}^{\bar{\phi}}\right)\right]+2 \Omega_{b}\left[\bar{r}\left(\xi_{, \bar{r}}^{i} \delta_{j}^{\bar{\phi}}-\delta_{\bar{r}}^{i} \xi_{, j}^{\bar{\phi}}\right)+\bar{r}^{-1}\left(\delta_{\bar{\phi}}^{i} \xi_{, j}^{\bar{r}}-\xi_{, \bar{\phi}}^{i} \delta_{j}^{r}\right)-\bar{r}^{-1} \xi^{\bar{r}}\left(\bar{r}^{-1} \delta_{\bar{\phi}}^{i} \delta_{j}^{\bar{r}}+\bar{r} \delta_{\bar{r}}^{i} \delta_{j}^{\bar{\phi}}\right)\right]
$$


Next we choose a fiducial streamline $\bar{r}=\bar{r}_{0}, \bar{z}=0$ and introduce the pseudo-Cartesian coordinates

$$
x^{\prime} \equiv \bar{r}-\bar{r}_{0}, \quad y^{\prime} \equiv \bar{r}_{0}\left(\bar{\phi}-\bar{\phi}_{0}\right), \quad z^{\prime} \equiv \bar{z} .
$$

The origin of this coordinate system follows the undisturbed motion along the fiducial streamline, so

$$
\frac{d \bar{\phi}_{0}}{d t} \equiv \Omega\left(\bar{r}_{0}\right)-\Omega_{b}
$$

The shearing-sheet velocity components are

$$
\begin{aligned}
& v_{x}^{\prime} \equiv \bar{v}^{\bar{r}}, \\
& v_{y}^{\prime} \equiv \bar{r}_{0} \bar{v}^{\bar{\phi}}-\bar{r}_{0}\left[\Omega(\bar{r})-\Omega_{b}\right]=\bar{r}_{0}\left[\bar{v}^{\bar{\phi}}-\Omega\left(\bar{r}_{0}\right)+\Omega_{b}\right]-2 A x^{\prime}+O\left(x^{\prime 2}\right), \\
& v_{z}^{\prime} \equiv \bar{v}^{\bar{z}} .
\end{aligned}
$$

We have introduced the Oort "constant,"

$$
\left.A \equiv \frac{1}{2} \frac{d \Omega(\bar{r})}{d \ln \bar{r}}\right|_{\bar{r}_{0}},
$$

which measures the strength of differential rotation. Henceforth, $\Omega$ will mean $\Omega\left(\bar{r}_{0}\right)$ when written without an argument. Both $\Omega$ and $A$ are treated as constants in the shearing sheet.

The Euler equations are presumed to be satisfied in the stationary state $v_{x}^{\prime}=v_{y}^{\prime}=v_{z}^{\prime}=0$. We expand the equations about this state in powers of $h / r$ and retain the nonstationary terms at $O\left(\Omega^{2} h\right)$. The coordinates $x, y$, and $z$ are treated as $O(h)$. The velocities $v_{x}$, $v_{y}$, and $v_{z}$ are $O(\Omega h)$, and they vary on length scales $h$ and timescales $\sim \Omega^{-1}$. (Here and henceforth, the primes will be dropped whenever doing so will not cause confusion.) For the purposes of this expansion, the horizontal displacements $\left(\xi r, \bar{r} \xi^{\phi}\right)$ are $O(r)$, and their spatial derivatives are $O(1)$ (derivatives with respect to $\bar{\phi}$ being normalized by factors of $\bar{r}^{-1}$ ). The vertical displacement $\xi^{z}$ is assumed to vanish at the disk midplane, so we assume that $\xi^{z}$ can be written as $\bar{z} f(\bar{r}, \bar{\phi})$, where the function $f$ is $O(1)$ and varies on a horizontal scale of order $r$.

To $O\left(\Omega^{2} h\right)$, the shearing-sheet equations are

$$
\begin{aligned}
\frac{d}{d t} v_{x}-2 \Omega v_{y}+\partial_{x} w^{\prime} & =-2 \Omega\left(c_{x x} v_{x}+c_{x y} v_{y}\right)+\left(b_{x x} \partial_{x} w^{\prime}+b_{x y} \partial_{y} w^{\prime}\right) \\
\frac{d}{d t} v_{y}+2(\Omega+A) v_{x}+\partial_{y} w^{\prime} & =-2 \Omega\left(c_{y x} v_{x}+c_{y y} v_{y}\right)+\left(b_{y x} \partial_{x} w^{\prime}+b_{y y} \partial_{y} w^{\prime}\right) \\
\frac{d}{d t} v_{z}+\partial_{z} w^{\prime} & =-2 \Omega c_{z z} v_{z}+b_{z z} \partial_{z} w^{\prime}
\end{aligned}
$$

where

$$
\frac{d}{d t} \equiv \partial_{t}+v_{x} \partial_{x}+\left(v_{y}+2 A x\right) \partial_{y}+v_{z} \partial_{z}
$$

is the time derivative following the fluid.

In equations (24), the quantity $w^{\prime}$ represents the difference between the actual enthalpy and the enthalpy in the stationary equilibrium: $w^{\prime} \equiv w-w^{(0)}$. In the shearing sheet, the latter has the form

$$
w^{(0)}=f_{0}(t)+f_{2}(t) z^{2}
$$

where $f_{0}$ and $f_{2}$ are functions of time ( $\equiv$ orbital phase) only. The term in $z^{2}$ would be nonzero even in a circular disk because of the hydrostatic response to the vertical field of the primary; in fact, $f_{2} \rightarrow-\frac{1}{2} \Omega^{2}$ in that case. In a tidally distorted disk, there are additional contributions due to the variation of the disk thickness with orbital phase.

The coefficients $c_{i j}$ and $b_{i j}$ are dimensionless and linear in the displacements:

$$
\begin{aligned}
& c_{x x}=-r^{-1} \xi_{, \phi}^{r}-r \xi_{, r}^{\phi}+\sigma \xi_{, r, \phi}^{r}, \\
& c_{x y}=r\left(r^{-1} \xi^{r}\right)_{, r}+\sigma r^{-1} \xi_{, \phi, \phi}^{r}-(1+\sigma) \xi_{, \phi}^{\phi}, \\
& c_{y x}=r\left(r^{-1} \xi^{r}\right)_{, r}+\sigma r \xi_{, \phi, r}^{\phi}-(1-\sigma) \xi_{, \phi}^{\phi}, \\
& c_{y y}=(1+\sigma) r^{-1} \xi_{, \phi}^{r}+r \xi_{, r}^{\phi}+\sigma \xi_{, \phi, \phi}^{\phi}, \\
& c_{z z}=\sigma \xi_{, z, \phi}^{z}, \\
& b_{x x}=2 \xi_{, r}^{r}, \\
& b_{x y}=b_{y x}=r \xi_{, r}^{\phi}+r^{-1} \xi_{, \phi}^{r}, \\
& b_{y y}=2\left(\xi_{, \phi}^{\phi}+r^{-1} \xi^{r}\right), \\
& b_{z z}=2 \xi_{, z}^{z},
\end{aligned}
$$


where $\sigma \equiv\left(\Omega-\Omega_{b}\right) / \Omega$. These coefficients are to be evaluated at $(r, \phi)=\left(\bar{r}_{0}, \bar{\phi}_{0}\right)$ and treated as independent of $x, y$, and $z$. However, $c_{i j}$ and $b_{i j}$ are time-dependent because of the motion of $\bar{\phi}_{0}(t)$ through the tidal distortion; like the displacements themselves, they oscillate sinusoidally at the frequency $m_{\text {tide }}\left[\Omega\left(\bar{r}_{0}\right)-\Omega_{b}\right]$. As in Paper I $(\S 3.6)$, the displacements $\left(\zeta^{r}, \xi^{\phi}, \xi^{z}\right)$ were related to the tidal potential of the secondary, and all azimuthal Fourier components of the tidal potential except $m_{\text {tide }}=2$ were neglected.

The equation for conservation of mass in the barred system is

$$
\partial_{t} \rho+\left(\rho \bar{v}^{i}\right)_{; i}=\partial_{t} \rho+\bar{g}^{-1 / 2}\left(\bar{g}^{1 / 2} \rho \bar{v}^{i}\right)_{, i}=0
$$

where $\bar{g}$ is the determinant of the metric tensor $\bar{g}_{i j}$ or, equivalently, the reciprocal of the determinant of its inverse $\bar{g}^{i j}$ (eq. [17a]). It is convenient to absorb this factor by defining the coordinate density $\bar{\rho}$, the mass per coordinate volume:

$$
\bar{\rho} \equiv \bar{g}^{1 / 2} \rho \text {. }
$$

In terms of this variable, the continuity equation (27) becomes

$$
\partial_{t} \bar{\rho}+\left(\bar{\rho} \bar{v}^{i}\right)_{, i}=0
$$

In the shearing sheet, therefore,

$$
\partial_{t} \rho_{\mathrm{ss}}+\partial_{x}\left(\rho_{\mathrm{ss}} v_{x}\right)+\partial_{y}\left[\rho_{\mathrm{ss}}\left(v_{y}+2 A x\right)\right]+\partial_{z}\left(\rho_{\mathrm{ss}} v_{z}\right)=0
$$

where

$$
\rho_{\mathrm{ss}} \equiv \bar{r}_{0}^{-1} \bar{\rho}=\bar{r}_{0}^{-1} g^{1 / 2} \rho
$$

is the coordinate density of mass per unit $d x d y d z$. Equation (30) has the same form as it would for a shearing sheet derived from a circular disk; the term $2 A x$ arises because the undisturbed coordinate velocity $\left(\bar{v}^{\bar{\phi}}\right)^{(\mathrm{eq})}=\Omega(\bar{r})-\Omega_{b}$ depends upon $x \equiv \bar{r}-\bar{r}_{0}$. In the stationary tidally distorted disk, $\bar{\rho}$ and $\rho_{\mathrm{ss}}$ are constant along streamlines.

In order to preserve the form of equations (24) when $\rho$ is replaced by $\rho_{\mathrm{ss}}$, we replace the physical pressure with the quantity

$$
p_{\mathrm{ss}} \equiv r_{0}^{-1} \bar{g}^{-1 / 2} p
$$

so that $w=\gamma p_{\mathrm{ss}} /(\gamma-1) \rho_{\mathrm{ss}}$.

For the numerical work we require the momentum equations in conservative form:

$$
\begin{aligned}
& \partial_{t}\left(\rho_{\mathrm{ss}} v_{x}\right)+\partial_{x}\left(\rho_{\mathrm{ss}} v_{x} v_{x}\right)+\partial_{y}\left(\rho_{\mathrm{ss}} v_{x} V_{y}\right)+\partial_{z}\left(\rho_{\mathrm{ss}} v_{x} v_{z}\right)+\partial_{x} p_{\mathrm{ss}}=S_{x}, \\
& \partial_{t}\left(\rho_{\mathrm{ss}} v_{y}\right)+\partial_{x}\left(\rho_{\mathrm{ss}} v_{y} v_{x}\right)+\partial_{y}\left(\rho_{\mathrm{ss}} v_{y} V_{y}\right)+\partial_{z}\left(\rho_{\mathrm{ss}} v_{y} v_{z}\right)+\partial_{y} p_{\mathrm{ss}}=S_{y}, \\
& \partial_{t}\left(\rho_{\mathrm{ss}} v_{z}\right)+\partial_{x}\left(\rho_{\mathrm{ss}} v_{z} v_{x}\right)+\partial_{y}\left(\rho_{\mathrm{ss}} v_{z} V_{y}\right)+\partial_{z}\left(\rho_{\mathrm{ss}} v_{z} v_{z}\right)+\partial_{z} p_{\mathrm{ss}}=S_{z},
\end{aligned}
$$

where

$$
V_{y} \equiv v_{y}+2 A x
$$

Equations (33) are obtained in the standard way: for example, to get equation (33a), we multiply equation (24a) by $\rho_{\text {ss }}$ and add this to $v_{x}$ times equation (30). The source terms on the right-hand side represent centrifugal and Coriolis forces, the gravitational field of the primary, differential rotation, and the shear and vorticity associated with the noncircularity of the stationary streamlines:

$$
\begin{aligned}
& S_{x}=2 \Omega \rho_{\mathrm{ss}} v_{y}-2 \Omega\left(c_{x x} \rho_{\mathrm{ss}} v_{x}+c_{x y} \rho_{\mathrm{ss}} v_{y}\right)+\left(b_{x x} \partial_{x} p_{\mathrm{ss}}+b_{x y} \partial_{y} p_{\mathrm{ss}}\right), \\
& S_{y}=-2(A+\Omega) \rho_{\mathrm{ss}} v_{x}-2 \Omega\left(c_{y x} \rho_{\mathrm{ss}} v_{x}+c_{y y} \rho_{\mathrm{ss}} v_{y}\right)+\left(b_{y x} \partial_{x} p_{\mathrm{ss}}+b_{y y} \partial_{y} p_{\mathrm{ss}}\right), \\
& S_{z}=\rho_{\mathrm{ss}} \partial_{z} w^{(0)}-2 \Omega c_{z z} \rho_{\mathrm{ss}} v_{z}+b_{z z}\left(\partial_{z} p_{\mathrm{ss}}-\rho_{\mathrm{ss}} \partial_{z} w^{(0)}\right) .
\end{aligned}
$$

In order to derive an energy equation, we first need an equation for the pressure. Under adiabatic conditions, $p \propto \rho^{\gamma}$, whence

$$
p_{\mathrm{ss}} \propto\left(r_{0}^{-1} \bar{g}^{1 / 2}\right)^{1-\gamma} \rho_{\mathrm{ss}}^{\gamma} .
$$

In differential form, with the use of equation (30), this becomes

$$
\frac{d p_{\mathrm{ss}}}{d t}+\gamma p_{\mathrm{ss}}\left(\partial_{x} v_{x}+\partial_{y} v_{y}+\partial_{z} v_{z}\right)=-(\gamma-1) p_{\mathrm{ss}} \frac{d}{d t}\left(\ln \bar{g}^{-1 / 2}\right)
$$

The term on the right-hand side is a nuisance, because it causes $p_{\text {ss }}$ to be nonconstant even in the stationary solution. We have neglected this term in our calculations. This was done by mistake, but we argue that the term is unimportant in any case. Clearly, the term vanishes in the isothermal limit $(\gamma \rightarrow 1)$. Less obviously, the term has no effect in the incompressible limit $(\gamma \rightarrow \infty)$ either, because the pressure is then not a dynamical variable but is determined indirectly by the constraint $\nabla \cdot v=0$. In fact, this term is unimportant even for intermediate values of $\gamma$ as long as the perturbed motions are strongly subsonic (as they proved to be, even in the nonlinear phases of our simulations), because the motions are then effectively incompressible. What this term does is to modulate the enthalpy and the sound speed by a spatially constant but temporally sinusoidal factor; however, the effects of this modulation are $O\left[\left(v / c_{s}\right)^{2}\right]$ compared with the dominant destabilizing terms in the source terms (eq. [35]).

The energy equation now follows by multiplying equation (33a), (33b), and (33c) by $v_{x}, v_{y}$, and $v_{z}$, respectively, adding these to the result of multiplying equations (24a), (24b), and (24c) by $\rho_{\mathrm{ss}} v_{x}, \rho_{\mathrm{ss}} v_{v}$, and $\rho_{\mathrm{ss}} v_{z}$, respectively; dividing by 2 ; and invoking the pressure 
equation (36):

$$
\begin{aligned}
\partial_{t} E+\partial_{x}\left[\left(E+p_{\mathrm{ss}}\right) v_{x}\right]+\partial_{y}\left[\left(E+p_{\mathrm{ss}}\right) V_{y}\right]+\partial_{z}\left[\left(E+p_{\mathrm{ss}}\right) v_{z}\right] \\
=S_{x} v_{x}+S_{y} v_{y}+S_{z} v_{z}-\left[p_{\mathrm{ss}} \frac{d}{d t}\left(\ln \bar{g}^{-1 / 2}\right)\right] \\
\quad \equiv S_{E}
\end{aligned}
$$

where

$$
E \equiv \frac{p_{\mathrm{ss}}}{\gamma-1}+\frac{1}{2} \rho_{\mathrm{ss}}\left(v_{x}^{2}+v_{y}^{2}+v_{z}^{2}\right)
$$

and the term in square brackets on the second line of equation (37) derives from the right-hand side of equation (36).

Derivatives of the fluid variables with respect to $y$ (the quasi-azimuthal coordinate along the stationary streamlines) were neglected in this paper because, as explained in $\S 1$, the linearly unstable modes vary much more rapidly in $\bar{r}$ and $\bar{z}$ than in $\phi$. This need not be true of the nonlinear disturbances into which the modes develop, but by placing this restriction on the nonlinear behavior, we reduce the computational problem from three to two spatial dimensions.

\subsection{Mass and Angular Momentum Fluxes}

In $\S 4$ we will want to measure the mass and angular momentum fluxes transported by turbulence when the instability becomes nonlinear. We require expressions for these fluxes in terms of the fluid variables.

In thin circular disks, the local mass flux in the cylindrical $r$ direction is $\rho v^{r}$. The phrase "mass flux" sometimes refers to the integral of the local flux over a cylinder of constant $r$; in this sense, the phrase is synonymous with the mass accretion rate at $r$,

$$
\dot{M}_{\text {circ }}(r)=\int_{0}^{2 \pi} d \phi \int_{-\infty}^{\infty} d z r \rho v^{r}
$$

Note the factor $r=(\operatorname{det} g)^{1 / 2}=$ the physical volume associated with the coordinate volume $d r d \phi d z$ (where $d r \rightarrow v^{r} d t$, since we are dealing with the flux along $r$ ).

It is desirable to define the local mass flux in our tidally distorted disks in such a way that the flux vanishes when the disk is stationary and the streamlines closed. This condition is satisfied by the component of the flux in the $\bar{r}$ direction. Hence we define the local mass flux, including the metric factor, as

$$
F^{(m)} \equiv r_{0}^{-1}(\operatorname{det} \bar{g})^{1 / 2} \rho \bar{v}^{\bar{r}}=\rho_{\mathrm{ss}} v_{x},
$$

in which the final expression is the form appropriate to the shearing sheet. As defined, $F^{(m)}$ is the flux of mass per unit coordinate area $d y d z$, rather than physical area $r_{0}^{-1} \bar{g}^{-1 / 2} d y d z$.

The mass accretion rate should be the integral of equation (40) over $y$ and $z$. The azimuthal integral, however, is replaced by an integral over time - or, rather, orbital phase - since the $y$ coordinate is restricted to a range $\ll \bar{r}_{0}$. This replacement is actually justified only if the turbulent motions are statistically stationary; that is, the dynamics of the region we have chosen to define our shearing sheet, when averaged over a few orbits, should be typical of those in other regions at the same $\bar{r}$.

We also require an expression for the angular momentum flux. By this we shall mean the component of that quantity in the $\bar{r}$, rather than $r$, direction. As in the theory of circular disks, we divide the angular-momentum flux into two parts: a part carried bodily by the mass at the mean radial velocity $\left(\bar{v}^{\bar{r}}\right)$; and a part carried by fluctuating velocities (with vanishing mean), pressure gradients, magnetic fields, etc. The first we shall call the "advective" flux, and the second, for lack of a better term, the "viscous" flux-even though no true microscopic viscosity need be involved. In the classical steady state thin disk, the advective and viscous fluxes are nearly equal and opposite; the normally positive (= outward) viscous flux allows mass accretion and its associated advective flux to proceed without rapid accretion of angular momentum. We are interested here in the viscous part of the flux. After writing out an expression for the total flux, we shall discard those terms that can be identified with the advective flux.

The angular momentum of the fluid per unit mass is

$$
\begin{aligned}
j & =r^{2}\left(v^{\phi}+\Omega_{b}\right) \\
& =\left(\bar{r}+\xi^{r}\right)^{2}\left(\bar{v}^{\bar{\phi}}+\Omega_{b}+\xi^{\phi}{ }_{, r} \bar{v}^{\bar{r}}+\xi^{\phi}{ }_{, \phi} \bar{v}^{\bar{\phi}}\right) .
\end{aligned}
$$

In the second line we have used relations (15) to express $j$ in the barred coordinates. The total angular momentum flux is

$$
F^{(j, \text { tot })}=r_{0}^{-1}(\operatorname{det} \bar{g})^{1 / 2}\left(\rho \bar{v}^{\bar{r}} j-p \partial_{\phi} \xi^{r}\right)=\left(\rho_{\mathrm{ss}} \bar{v}^{\bar{r}} j-p_{\mathrm{ss}} \partial_{\phi} \xi^{r}\right) \text {. }
$$

The pressure occurs here because the normal to a surface of constant $\bar{r}, \nabla \bar{r}$, has a component $\left(-r^{-1} \partial_{\phi} \xi^{r}\right)$ in the $\phi(\neq \bar{\phi})$ direction, and therefore the pressure exerted on such a surface transmits a torque from streamlines interior to $\bar{r}$ to those exterior. Using equation (41), we expand equation (42) to first order in the displacements, introduce the shearing-sheet velocities in equation (22), and evaluate at $\bar{r}=\bar{r}_{0}(x=0)$; this yields the "viscous" flux:

$$
F^{(j)}=\left(1+2 \bar{r}_{0}^{-1} \xi^{r}+\xi_{, \phi}^{\phi}\right) \rho_{\text {ss }} v_{x} v_{y}+\left(\bar{r}_{0} \xi_{, r}^{\phi}\right) \rho_{\text {ss }} v_{x}^{2}-\left(\bar{r}_{0}^{-1} \xi_{, \phi}^{r}\right) p_{\text {ss }} .
$$

An overall factor of $\bar{r}_{0}$ has simply been omitted, so that the quantity (43) actually has the dimensions of a momentum flux rather than an angular momentum flux: in the Cartesian coordinates of the shearing sheet, it is convenient to pretend that one deals with 
linear momentum rather than angular momentum, because this puts motions along $x$ and $y$ on the same footing. The advective terms that have been subtracted in passing from equation (42) to equation (43) are

$$
F^{(j, \text { adv })}=\left[\Omega \bar{r}_{0}+\left(\Omega-\Omega_{b}\right) \bar{r}_{0} \xi_{, \phi}^{\phi}+2 \Omega \xi^{r}\right] F^{(m)} .
$$

The final form in equation (43) has the awkward feature that the term in $p_{\text {ss }}$ gives rise to a nonzero local flux even in the stationary disk; however, since $\xi^{r},{ }_{\bar{\phi}}$ is antisymmetric about the axis between the two stars, and $p_{\text {ss }}$ is symmetric (or constant, if the right-hand side of eq. [36] is neglected), this term averages to zero over orbital phase in the stationary disk.

\section{COMPUTATIONAL METHOD}

For the moment, we retreat from the shearing sheet and consider compressible fluid dynamics in inertial, Cartesian coordinates.

\subsection{TVD Hydrodynamics}

The three-dimensional hydrodynamic equations of an ideal gas are

$$
\begin{gathered}
\partial_{t} \boldsymbol{q}+\partial_{x} \boldsymbol{F}_{x}+\partial_{y} \boldsymbol{F}_{y}+\partial_{z} \boldsymbol{F}_{z}=0, \\
\boldsymbol{q}=\left(\begin{array}{c}
\rho \\
\rho v_{x} \\
\rho v_{y} \\
\rho v_{z} \\
E
\end{array}\right), \quad \boldsymbol{F}_{x}=\left(\begin{array}{c}
\rho v_{x} \\
\rho v_{x}^{2}+p \\
\rho v_{x} v_{y} \\
\rho v_{x} v_{z} \\
(E+p) v_{x}
\end{array}\right), \quad \boldsymbol{F}_{y}=\left(\begin{array}{c}
\rho v_{y} \\
\rho v_{x} v_{y} \\
\rho v_{y}^{2}+p \\
\rho v_{y} v_{z} \\
(E+p) v_{y}
\end{array}\right), \quad \boldsymbol{F}_{z}=\left(\begin{array}{c}
\rho v_{z} \\
\rho v_{x} v_{z} \\
\rho v_{y} v_{z} \\
\rho v_{z}^{2}+p \\
(E+p) v_{z}
\end{array}\right), \\
E=\frac{p}{\gamma-1}+\frac{1}{2} \rho\left(v_{x}^{2}+v_{y}^{2}+v_{z}^{2}\right),
\end{gathered}
$$

where $\gamma$ is the adiabatic index. From the state vector, $\boldsymbol{q}$, and the flux functions, $\boldsymbol{F}_{\boldsymbol{x}}(\boldsymbol{q}), \boldsymbol{F}_{y}(\boldsymbol{q})$, and $\boldsymbol{F}_{z}(\boldsymbol{q})$, one can form the Jacobian matrices, $\boldsymbol{A}_{x}(\boldsymbol{q}) \equiv \partial \boldsymbol{F}_{x} / \partial \boldsymbol{q}, \boldsymbol{A}_{y}(\boldsymbol{q})=\partial \boldsymbol{F}_{y} / \partial \boldsymbol{q}$, and $\boldsymbol{A}_{z}(\boldsymbol{q})=\partial \boldsymbol{F}_{z} / \partial \boldsymbol{q}$. The system of equation (45) is hyperbolic, if all of the eigenvalues of each Jacobian matrix are real and the corresponding set of right eigenvectors is complete, which is true when $\boldsymbol{q}$ and $\boldsymbol{F}_{\boldsymbol{x}, \boldsymbol{y}, \boldsymbol{z}}$ are defined by equations (46).

The Total-Variation-Diminishing (TVD) scheme devised by Harten (1983) is an explicit, second-order Eulerian finite-difference scheme that solves such hyperbolic systems of conservation equations. TVD modifies the flux function and then applies a nonoscillatory first-order scheme to achieve second-order accuracy. The TVD method combines the high resolution of second-order spatial accuracy with the robustness of the nonoscillatory first-order scheme (it will not, for example, produce unphysical oscillations behind shocks and other discontinuities). Also, the TVD scheme requires less programming effort and less CPU time than other high-accuracy methods.

Harten described the application of his TVD scheme to one-dimensional hydrodynamics. Multidimensional versions of the TVD code can be obtained straightforwardly by Strang-type dimensional splitting (Strang 1968). In Appendix A we reproduce the eigenvalues and eigenvectors for the three-dimensional hydrodynamic equations, and in Appendix B we outline the dimensionalsplitting procedure.

The original TVD scheme was designed to capture shocks accurately, and Harten (1983) discusses its performance in shock-tube tests. This paper, however, involves purely subsonic flows for which shocks are not an issue. The TVD code has therefore been modified as described in Appendix B to minimize its numerical viscosity.

Our three-dimensional TVD code runs at the speed of $\sim 8 \times 10^{3}$ zones per second with one CPU on a Convex 220 and at $\sim 5 \times 10^{4}$ zones per second with one CPU on a Cray 2 .

\subsection{Numerical Viscosity}

Although we do not introduce any explicit viscous terms in the numerical equations, diffusion of momentum across cell boundaries is unavoidable and amounts to an effective numerical viscosity. Diffusion of energy also occurs and produces a numerical thermal conductivity, but the conductivity is small compared with the viscosity; even if it were not, it would have little effect on our subsonic flows.

To measure the numerical viscosity, we have compared the decay rate of simulated two-dimensional sound waves and shear flows with the predicted rate in a viscous fluid. The momentum and pressure equations for a viscous fluid can be written in nonconservative form as

$$
\begin{gathered}
\partial_{t} v_{i}+v \cdot \nabla v_{i}+\frac{1}{\rho} \partial_{i} p=\frac{1}{\rho} \partial_{k} \sigma_{i k} \\
\partial_{t} p+v \cdot \nabla p+\gamma p \nabla \cdot v=(\gamma-1) \sigma_{i k} \partial_{k} v_{i}
\end{gathered}
$$

In these equations,

$$
\sigma_{i k}=\eta\left(\partial_{k} v_{i}+\partial_{i} v_{k}-\frac{2}{3} \delta_{i k} \nabla \cdot v\right)+\zeta \delta_{i k} \nabla \cdot v
$$

is the viscosity tensor, where $\eta$ and $\zeta$ are the dynamic shear and bulk viscosity, respectively. 
We have calculated the damping of a diagonally propagating sound wave with initial conditions

$$
\delta v_{x}=\delta v_{y}=v_{\text {amp }} c_{s} \sin \left[\frac{2 \pi}{L}(x+y)\right], \quad \delta \rho=\delta p=0,
$$

where $c_{s}$ is the background sound speed. We chose $v_{\text {amp }}=10^{-4}$ and imposed periodic boundary conditions in both $x$ and $y$ with period $L$.

The predicted frequency of the wave is

$$
\omega=i\left(\frac{4}{3} \frac{\eta}{\rho_{0}}+\frac{\zeta}{\rho_{0}}\right) \frac{4 \pi^{2}}{L^{2}} \pm c_{s} \frac{2 \sqrt{2} \pi}{L} \sqrt{1-\frac{1}{c_{s}^{2}}\left(\frac{4}{3} \frac{\eta}{\rho_{0}}+\frac{\zeta}{\rho_{0}}\right)^{2} \frac{4 \pi^{2}}{L^{2}}}
$$

where $\rho_{0}$ is the background density. The damping rate, $\Gamma_{\text {decay }}$, is the imaginary part of $\omega$. For easier comparison of this test with the shear-flow test described below, we define a "Reynolds number" by

$$
R_{\text {sound wave }} \equiv c_{s} L /\left(\frac{4 \eta}{3 \rho_{0}}+\frac{\zeta}{\rho_{0}}\right) .
$$

Our second test involved a planar shear flow with initial conditions

$$
\delta v_{x}=\delta v_{y}=v_{\text {amp }} c_{s} \sin \left[\frac{2 \pi}{L}(x-y)\right], \quad \delta \rho=\delta p=0,
$$

again with $v_{\text {amp }}=10^{-4}$ and with the same periodic boundary conditions. This flow has an inflection point and is theoretically unstable to Kelvin-Helmholtz modes, but the minimum growth time is $\sim L / c_{s} v_{\text {amp }}$, which is much longer than the duration of our simulations; furthermore, the unstable streamwise wavelengths are larger than our computational domain. Therefore, the flow in equation (54) is predicted to decay viscously at the rate

$$
\Gamma_{\text {decay }}=\frac{2 \eta}{\rho_{0}} \frac{4 \pi^{2}}{L^{2}}
$$

and define the Reynolds number by

$$
R_{\text {shear flow }} \equiv \frac{c_{s} L \rho_{0}}{2 \eta}
$$

In Figure 1 the decay rates and Reynolds numbers for both tests are plotted as a function of the number of cells spanning the length $L$. Good fits of the form $\Gamma_{\text {decay }} \propto n_{\text {cells }}^{-2}$ and $R \propto n_{\text {cells }}^{2}$ are clearly possible, confirming that our code has second-order accuracy. Laboratory turbulence tends to be strongly damped if $R \lesssim 10^{3}$ (Reynolds 1883). The plot therefore indicates that our code can represent turbulent motions if they span more than $\sim 10$ cells.

\subsection{Elliptical Instability}

As a further test of our code, we have applied it to the Pierrehumbert-Bayly elliptical instability (Pierrehumbert 1986; Bayly 1986; Craik \& Criminale 1986). In its ideal form, this is a three-dimensional instability of an infinite, inviscid, incompressible, homoge-
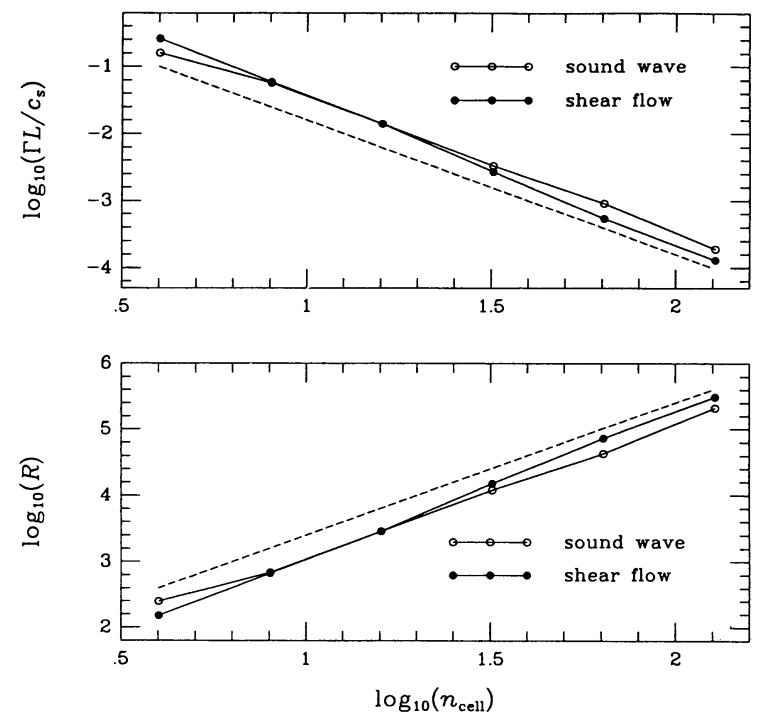

Fig. 1.-Normalized decay rate $\left(\Gamma_{\text {decay }} L / c_{s}\right)$ and Reynolds number $(R)$ vs. resolution (the number of cells spanned by the box size $L$ ) in the tests of twodimensional sound wave and sinusoidal planar shear flow. For comparison, dashed lines with $\left(\Gamma_{\text {decay }} L / c_{s}\right) \propto n_{\text {cell }}^{-2}$ and $R \propto n_{\text {cell }}^{2}$ are drawn. 
neous fluid whose unperturbed velocity field is a linear function of position,

$$
\boldsymbol{v}(\boldsymbol{r})=\boldsymbol{A} \cdot \boldsymbol{r},
$$

with closed elliptical streamlines. A review and physical interpretation of this instability is given in Paper I. It is closely related to the disk instabilities studied here and in our previous paper. Because the elliptical instability can be observed in a flow that is not geometrically thin, however, we can forgo the local shearing-sheet approximation and its attendant source terms.

Since our code is limited to finite rectangular grids, we simulated the velocity field

$$
\begin{aligned}
& v_{x}=-\frac{1}{4} \cos [\pi(x-0.5)] \sin [2 \pi(y-0.25)], \\
& v_{y}=\frac{1}{8} \sin [\pi(x-0.5)] \cos [2 \pi(y-0.25)], \\
& v_{z}=0,
\end{aligned}
$$

in the region $0 \leq x \leq 1,0 \leq y \leq 0.5,0 \leq z \leq 0.5$, with reflecting freely slipping boundary conditions. The initial density and pressure are set to constant values, and units are chosen such that the initial sound speed is unity. At every grid point we add random noise at the level $\left|\delta v_{i}\right| \leq 10^{-6}$ to each component of the velocity. Note that the flow in equations (58) is not in equilibrium, because no pressure gradient has been set up to balance the centrifugal forces. Furthermore, the fluid simulated by our code is compressible. However, since the maximum velocity in equations $(58)$ is substantially subsonic $\left[\left(v / c_{s}\right)_{\max }=0.25\right]$, the flow executes rather small-amplitude pulsations around its initial form until the instability develops.

The central part of the flow field in equations (58) approximates an incompressible elliptical vortex of the form of equation (57),

$$
v=\Omega_{E}\left[-E(y-0.25), \frac{x-0.5}{E}, 0\right],
$$

with $\Omega_{E}=\pi / 4$ and $E=2$. According to the linear analysis of Bayly (1986), the maximum growth rate at this ellipticity is $\bar{\Gamma}_{E}=$ $0.38 \Omega_{E}$.

Figure 2 shows the time evolution of the root mean square of each velocity component in the linear regime $(0 \leq t \leq 80)$ of a simulation carried out on a $100 \times 50 \times 50$ grid in $x y z$. The curve for $\mathrm{J} v_{z}^{2} \mathrm{~N}^{1 / 2}$ shows exponential growth at the rate $\bar{\Gamma}_{E} / \Omega_{E} \sim 0.27$. A similar calculation with $64 \times 32 \times 32$ cells gives $\bar{\Gamma}_{E} / \Omega_{E} \sim 0.22$, and one with $32 \times 16 \times 16$ cells gives $\bar{\Gamma}_{E} / \Omega_{E} \sim 0.14$. As pointed out in Landman \& Saffman (1987), viscosity will reduce the growth rate. In the limit of small ellipticity $(E \rightarrow 1)$, the growth rate of perturbations with wavenumber $\boldsymbol{k}$ is decreased by $\left(\eta / \rho_{0}\right) k^{2}$. From the previous section, the numerical viscosity in our code is proportional to the cell size squared. Extrapolating the numerically obtained growth rates cited above to zero cell size, we obtain $\bar{\Gamma}_{E} / \Omega_{E} \sim 0.3$. This is somewhat smaller than the growth rate $\bar{\Gamma}_{E} / \Omega_{E} \sim 0.38$ predicted by the linear analysis for an $E=2$ elliptical flow.

We have performed a similar calculation with larger noise added to the initial velocity field of equation (58), and we have pursued the evolution well past the end of exponential growth. Figure 3 shows a horizontal slice through the velocity field in the initial state and at three later times: $t=\mathbf{4 0}$ marks the end of the linear phase. The instability develops first near the center of the vortex where the shear $\left|\partial_{y} v_{x}+\partial_{x} v_{y}\right|$ is largest. In the nonlinear phase, the vortex disrupts into smaller eddies, and its energy and vorticity are transferred to small scales, where they are rapidly damped by numerical viscosity. At $t=60$, about $45 \%$ of the initial kinetic energy has been converted to internal energy, and the remainder is equally divided among the three Cartesian directions.

To verify that the instability is truly three-dimensional, we have repeated the calculation shown in Figure 3 on a single horizontal plane (that is, $100 \times 50 \times 1$ instead of $100 \times 50 \times 50$ ). In this case, essentially nothing happens: the initial vortex persists virtually unchanged and its energy has decreased by only a few percent at $t=60$, presumably as a result of numerical viscosity.

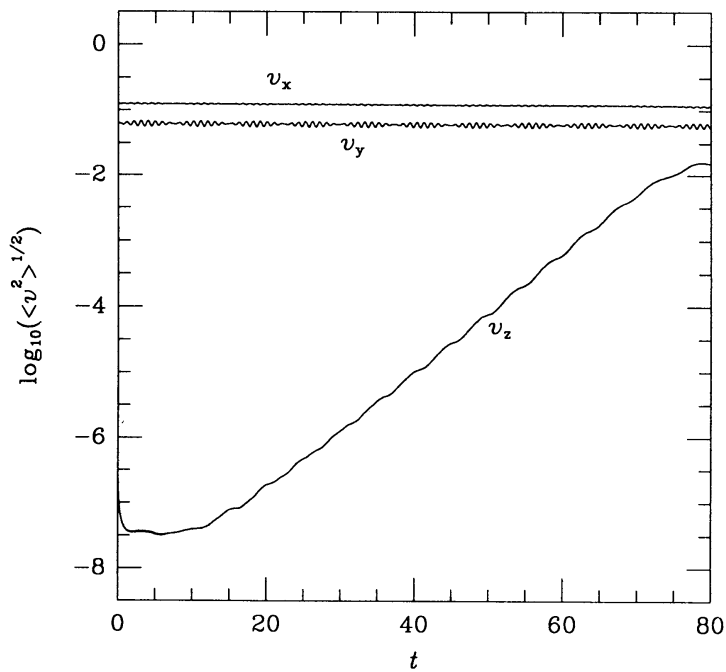

FIG. 2.-Spatially averaged rms velocity components vs. time for the low-initial-noise simulation of the elliptical vortex (eq. [58]). See text for details. 

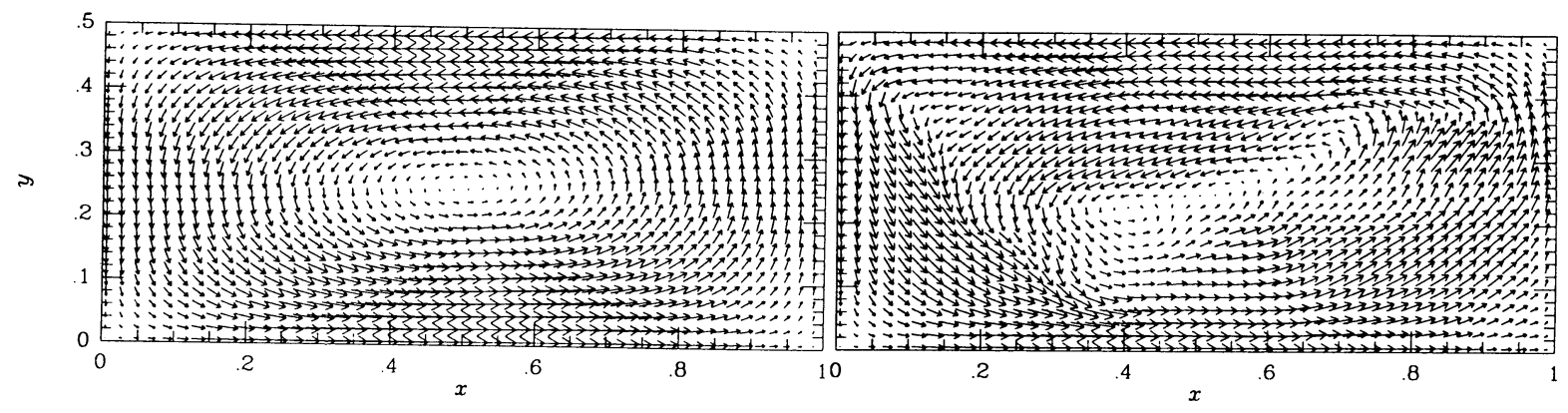

$t=40$

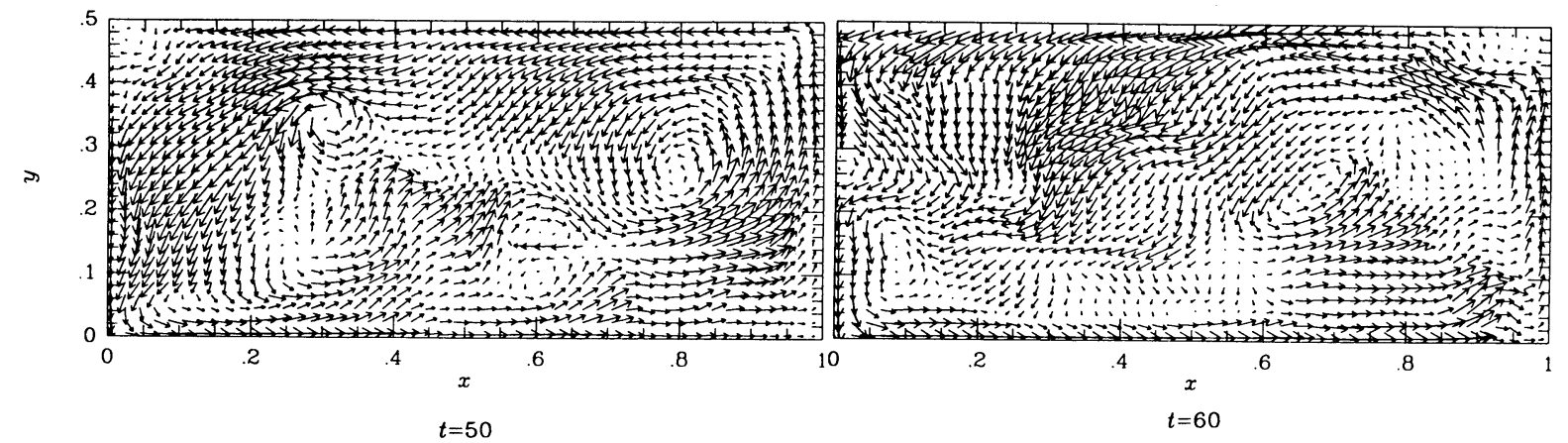

Fig. 3.-Horizontal velocity field on the 26th horizontal plane of the high-initial-noise simulation of the elliptical instability. The top left-hand panel shows the initial conditions (58) after a brief adjustment period during which small-scale noise damps out; times of remaining panels are marked.

\section{LOCAL SIMULATIONS OF TIDALLY DISTORTED ACCRETION DISKS}

In this section, we shall omit the subscript "ss" from $\rho$ and $p$, but it should be remembered that these quantities differ at $O(\xi / r)$ from the physical density and pressure.

\subsection{Boundary Conditions, Units, and Model Parameters}

We have applied our TVD code to the shearing-sheet equations (30)-(38) with the simplifications noted in $\S 2$. That is, we neglect all derivatives with respect to the $y$ coordinate, but all three components of velocity are included. The source terms in equations (35) have been incorporated in such a way as to preserve second-order accuracy; details are given in Appendix C.

We have neglected the vertical structure of the unperturbed disk by dropping the terms involving $\partial_{z} w^{(0)}$, the vertical derivative of the unperturbed enthalpy, from the source terms in equations (35). On the one hand, linear analysis indicates that these terms are not important to the growth rate except near the radius at which

$$
m^{2}\left(\Omega-\Omega_{b}\right)^{2}=(\gamma+1) \Omega^{2},
$$

where there is a direct resonance between a vertical pulsation mode and the tidal forcing (see Paper I). On the other hand, these terms introduce numerical complications in the vertical boundary condition.

We use periodic boundary conditions in the radial (x) direction. This means, in particular, that $v_{y}\left(x+L_{x}\right)=v_{y}(x)$, where $L_{x}$ is the periodicity length (and similarly for $v_{x}, v_{z}, \rho$, and $p$ ). This boundary condition is not inconsistent with differential rotation of the stationary background flow: the total "azimuthal " contravariant velocity component in the streamline coordinates is

$$
\bar{v}^{\bar{\phi}}=r_{0}^{-1}\left(v_{y}+2 A x\right)+\Omega\left(r_{0}\right)-\Omega_{b},
$$

which is not periodic in $x$. In the vertical (z) direction, we adopt reflecting boundary conditions, $v_{z}=\partial_{z} v_{x}=\partial_{z} v_{y}=\partial_{z} p=\partial_{z} \rho=0$. We expect that these relatively low-frequency motions should be reflected at the surface of a real disk. Although the computational domain is smaller than the disk scale height $(H)$ in most of our simulations, we believe that it is potentially important for the nonlinear saturation that the linear modes should be standing (rather than traveling) waves in $z$ as well as radius $(x)$.

The dimensional units of our simulations have been defined by setting the following quantities to unity: the background density $\rho_{0}$, the length $L$ of one side of the (square) computational domain, and the local orbital frequency $\Omega$. The adiabatic index $\gamma$ has been set to $5 / 3$, and the Oort constant $A$ to $-(3 / 4) \Omega$, as appropriate for a Keplerian disk; therefore, $\kappa \equiv[4 \Omega(\Omega+A)]^{1 / 2}=\Omega$.

The description of the stationary background is completed, apart from parameters of the tidal distortion, by specifying the background pressure, $p_{0}$, or equivalently the background sound speed, $c_{s}=\left(\gamma p_{0} / \rho_{0}\right)^{1 / 2}$. As we increase $c_{s} / L \Omega$, the fluid behaves more and more nearly incompressibly. We expect that $c_{s}$ should be irrelevant to the modes of interest to us in the limit $c_{s} / L \Omega \rightarrow \infty$, but we cannot take that limit in our explicit TVD scheme. The sound speed is also related to the vertical scale height, $c_{s} \sim \Omega H$. Thus $H / L \propto c_{s} / L \Omega$, and therefore, in our units, large $c_{s}$ corresponds to computational domains that are small compared with the scale height. 
TABLE 1

\begin{tabular}{crccc}
\multicolumn{5}{c}{ MODEL PARAMETERS } \\
\hline \hline Run & Mesh & $M_{2} / M_{1}$ & $r / a$ & $c_{s} / L \Omega$ \\
\hline $1 \ldots \ldots \ldots \ldots \ldots$. & $64^{2}$ & $1 / 5$ & 0.4 & 3 \\
$2 \ldots \ldots \ldots \ldots \ldots$. & $128^{2}$ & $1 / 5$ & 0.4 & 3 \\
$3 \ldots \ldots \ldots \ldots \ldots$. & $64^{2}$ & $3 / 20$ & 0.4 & 3 \\
$4 \ldots \ldots \ldots \ldots \ldots$. & $128^{2}$ & $3 / 20$ & 0.4 & 3 \\
$5 \ldots \ldots \ldots \ldots \ldots$. & $64^{2}$ & $1 / 10$ & 0.4 & 3 \\
$6 \ldots \ldots \ldots \ldots \ldots$. & $64^{2}$ & $2 / 30$ & 0.4 & 3 \\
$7 \ldots \ldots \ldots \ldots \ldots$. & $64^{2}$ & $1 / 5$ & 0.4 & 1 \\
$8 \ldots \ldots \ldots \ldots \ldots$ & $64^{2}$ & $1 / 5$ & 0.4 & 5 \\
\hline
\end{tabular}

The linear growth rate of the elliptical instability depends on strength of the tidal distortion, which in turn depends upon the mass ratio $M_{2} / M_{1}$ and upon $r_{0} / a$, where $a=r_{2}$ is the binary semimajor axis. The values of these parameters for each of our simulations are listed in Table 1.

According to the considerations of $\S 1$ and Paper I, parametric destabilization of the inertial oscillations is strongest when their wavenumber satisfies

$$
k_{z}^{2}=k_{x}^{2} \frac{\left(\Omega-\Omega_{b}\right)^{2}}{\Omega^{2}-\left(\Omega-\Omega_{b}\right)^{2}}+\frac{\left(\Omega-\Omega_{b}\right)^{2}}{c_{s}^{2}},
$$

The final term is a correction due to compressibility and can be derived by replacing the dispersion relation (2) with

$$
\omega^{\prime 4}-\left(c_{s}^{2} k^{2}+\kappa^{2}\right) \omega^{\prime 2}+c_{s}^{2} k_{z}^{2} \kappa^{2}=0 .
$$

In the numerical simulations, the allowed values of $k_{x}$ and $k_{z}$ are limited by the dimensions of computational domain and by the number of cells used: if these are $L_{z} \times L_{z}$ and $n_{x} \times n_{z}$, then $k_{x} L_{x} /(2 \pi) \in\left\{0, \pm 1, \ldots, \pm\left(n_{x} / 2\right)\right\}$ and $k_{z} L_{z} / \pi \in\left\{0,1, \ldots, n_{z}\right\}$. We have chosen $r_{0} / a$, or equivalently $\left(\Omega-\Omega_{b}\right) / \Omega$, and $M_{2} / M_{1}$ so that $L_{z} / L_{x}=1$ is close to the ratio $k_{x} / k_{z}$ corresponding to the maximum growth rate in the incompressible limit. For the simulations listed in Table 1, the latter ratio lies between 0.912 (run 6 ) and 0.955 (run 8).

In column (2) of Table 2 we have listed the maximum growth rate predicted by Paper I; this prediction takes no account of compressibility or of the geometric constraints just discussed. We list in column (3) predictions derived from a direct linear analysis of the fluid equations (30)-(37) assuming $k_{x}=k_{z}=2 \pi / L$.

In each simulation, we have added a random component to $v_{z}$ uniformly distributed between $-10^{-2}$ and $10^{2}$ in order to initiate the instability.

Even though the somulations reported in this paper are only two-dimensional, they are still rather expensive in CPU time, since they were typically continued for several hundered sound-crossing times $\left(L / c_{s}\right)$. For instance, run 2 in Table 2 has been evolved to $t=200 / \Omega=600 L / c_{s}$, which requires $\sim 10^{5}$ computational steps. Including the calculation of statistics, this takes $\sim 50 \mathrm{CPU}$ hours on a Convex 220 and $\sim 8 \mathrm{CPU}$ hours on a Cray 2.

\subsection{Results}

Figure 4 shows the history of the root mean square perturbed velocities averaged over the grid for the first four runs listed in Table 2. After an initial adjustment, the velocity perturbations grow exponentially for a while and then level off. We shall refer to the exponential phase as the "linear" regime, and to the subsequent period as the "nonlinear" or "saturated" regime. The terminology seems justified because the structure of the flow is quite different before and after saturation, as can be seen by comparing the upper (linear regime) and lower (nonlinear regime) panels of Figure 5 . The quantity displayed in Figures $5 b$ and $5 d$ is actually $\rho v_{y}+2(\rho$ $\left.-\rho_{0}\right)(A+\Omega)(x-L / 2)$, which would be Eulerian perturbation in the angular momentum density (apart from a factor of $\left.r_{0}\right)$ if the disk were not tidally distorted; however, the true angular momentum density looks very similar to this, since the corrections are smaller than what is shown by $O\left(\xi / r_{0}\right)$. Similarly, $(\nabla \times v)_{y}$ is almost but not exactly the physical component of vorticity parallel to

TABLE 2

Growth Rate and Saturation Velocity

\begin{tabular}{cccccccc}
\hline \hline $\begin{array}{c}\text { Run } \\
(1)\end{array}$ & $\begin{array}{c}s_{\max } / \Omega \\
(2)\end{array}$ & $\begin{array}{c}s_{\text {lin }} / \Omega \\
(3)\end{array}$ & $\begin{array}{c}s_{\text {sim }} / \Omega \\
(4)\end{array}$ & $\begin{array}{c}\left(\delta \rho / \rho_{0}\right) /\left(\delta v / \delta c_{s}\right) \\
(5)\end{array}$ & $\begin{array}{c}k \delta v / s_{\max } \\
(6)\end{array}$ & $\begin{array}{c}\dot{E} /\left(s_{\max } \rho \delta c^{2}\right) \\
(7)\end{array}$ & $\begin{array}{c}t_{\text {interval }} \\
(8)\end{array}$ \\
\hline $1 \ldots \ldots \ldots \ldots$ & 0.139 & 0.136 & 0.135 & $1.33 \times 10^{-2}$ & 9.59 & 0.70 & $80 \leq t \Omega \leq 100$ \\
$2 \ldots \ldots \ldots \ldots$ & 0.139 & 0.136 & 0.135 & $1.16 \times 10^{-2}$ & 8.76 & 0.65 & $80 \leq t \Omega \leq 100$ \\
$3 \ldots \ldots \ldots \ldots$ & 0.101 & 0.097 & 0.085 & $1.19 \times 10^{-2}$ & 9.33 & 0.51 & $120 \leq t \Omega \leq 150$ \\
$4 \ldots \ldots \ldots \ldots$ & 0.101 & 0.097 & 0.093 & $1.07 \times 10^{-2}$ & 10.38 & 0.44 & $120 \leq t \Omega \leq 150$ \\
$5 \ldots \ldots \ldots \ldots$ & 0.065 & 0.057 & 0.053 & $1.00 \times 10^{-2}$ & 12.22 & 0.54 & $170 \leq t \Omega \leq 200$ \\
$6 \ldots \ldots \ldots \ldots$ & 0.043 & 0.027 & 0.026 & $8.02 \times 10^{-3}$ & 12.89 & 0.34 & $320 \leq t \Omega \leq 375$ \\
$7 \ldots \ldots \ldots \ldots$ & 0.139 & 0.128 & 0.126 & $1.35 \times 10^{-2}$ & 9.40 & 0.67 & $80 \leq t \Omega \leq 100$ \\
$8 \ldots \ldots \ldots \ldots$ & 0.139 & 0.136 & 0.130 & $5.70 \times 10^{-3}$ & 8.95 & 0.99 & $80 \leq t \Omega \leq 100$ \\
\hline
\end{tabular}




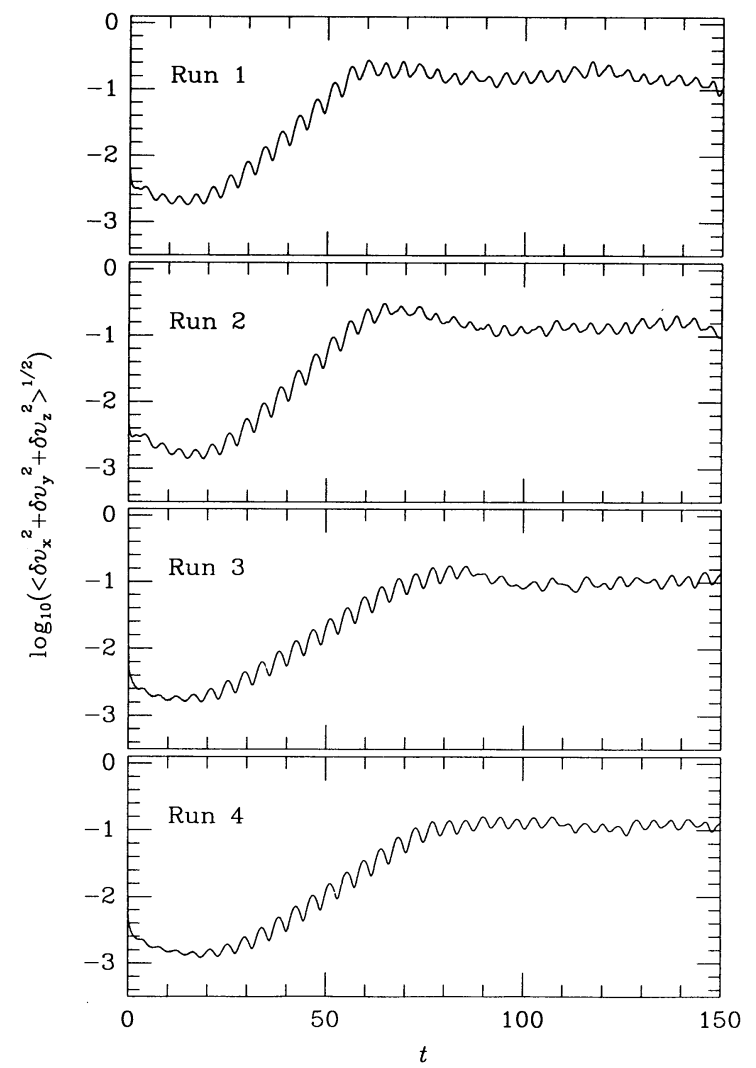

FIG. 4.-Time evolution of the spatially averaged rms velocity perturbations in runs 1-4 (Table 1)

the unperturbed streamlines. While the primary model $\left|k_{x}\right|=\left|k_{z}\right|=2 \pi / L$ dominates in the linear regime, the nonlinear structure looks quite chaotic. This is confirmed by the Fourier analysis reported below.

From the plots in Figure 4 and similar plots for the remaining runs, we have estimated the linear growth rates listed in column (4) of Table 2. These agree quite well with the growth rates in column (3) from the linear analysis. We conclude that our numerical code has successfully followed at least the linear development of the elliptical instability for the primary mode.

Column (5) of Table 2 lists the ratio, in the saturated regime, of the root mean square relative density perturbation, $\left\langle\delta \rho^{2}\right\rangle^{1 / 2} / \rho_{0}$, to the rms Mach number of the perturbations, $\delta v / c_{s} \equiv\left\langle v_{x}^{2}+v_{y}^{2}+v_{z}^{2}\right)^{1 / 2} / c_{s}$. (The averages are taken over the entire computational domain and over the time interval listed in col. [7].) Clearly, compressibility is a small effect, even when $L / H=1$. This distinguishes the instabilities studied here from those studied by Kaisig $(1989 \mathrm{a}, \mathrm{b})$, which developed as modified sound waves and saturated in weak shocks.

Heuristic arguments in Paper I predicted that, after saturation,

$$
\delta v \approx f_{1} \frac{s_{\max }}{k}
$$

where $s_{\max }$ is the local linear growth rate maximized with respect to the orientation of $k$, and $f_{1}$ is a dimensionless coefficient of order unity. Column (6) of Table 2 therefore displays $k \delta v / s_{\max }\left[k \equiv 2 \pi(2)^{1 / 2} / L\right]$. Evidently, this quantity is fairly constant, and $f_{1} \approx 9-13$. The success of the prediction in equation (61) may be due in part to the limited range in growth rate that we have studied [in fact, a better fit to these data is $\left.k \delta v / \Omega \propto\left(s_{\max } / \Omega\right)^{0.7}\right]$. There are some theoretical reasons to suspect that the scaling $(61)$ could fail completely at very small $s / \Omega-$ a regime that we are not able to simulate by the methods of this paper. Fortunately, relatively large values of $s / \Omega$ are predicted in the outer parts of accretion disks, where these instabilities are likely to have their greatest astrophysical importance (see $\S 5$ ).

Paper I also predicted that after saturation the fluid should absorb energy at the rate

$$
\dot{E}=f_{2} s_{\max } \delta v^{2}
$$

per unit mass, where $f_{2}$ is a second dimensionless factor of order unity. ${ }^{4}$ The argument here was that the nonlinear dissipation rate should balance the rate at which the energy of the primary model $\left(=\delta v_{\text {mode }}^{2} / 2\right)$ would increase if it continued to grow at the linear rate. It was implicitly assumed that the kinetic energy in the fluid at saturation was comparable to the energy in the primary mode alone (that is, $\delta v_{\text {mode }} \sim \delta v$ ). This is qualitatively supported by the steepness of the velocity power spectrum (see below), which implies

\footnotetext{
${ }^{4}$ The symbol $E$ was used earlier to signify an energy per unit volume. We shall shortly use $E_{\text {tot }}$ for the total energy of the disk. In each case, the dimensions of $E$ should be clear from the context.
} 


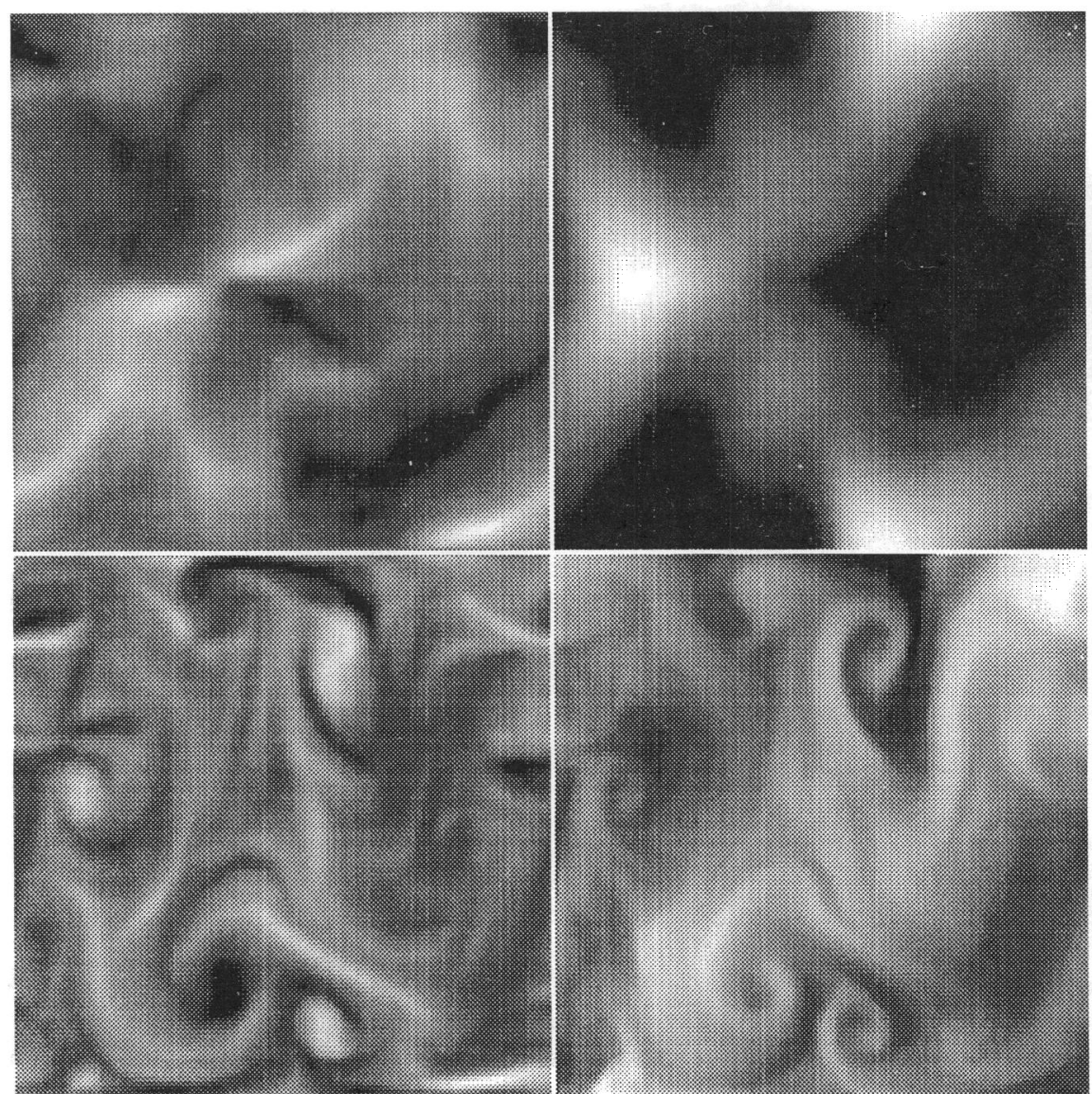

(c)

(d)

Fig. 5. - Gray-scale plots in the $(x, z)$-plane of two fluid variables in run 2. White is positive, and black is negative. $(a, c)(\nabla \times v)_{y}$ at $t=50,110 .(b, d)$ Angular momentum perturbation at $t=50,110$.

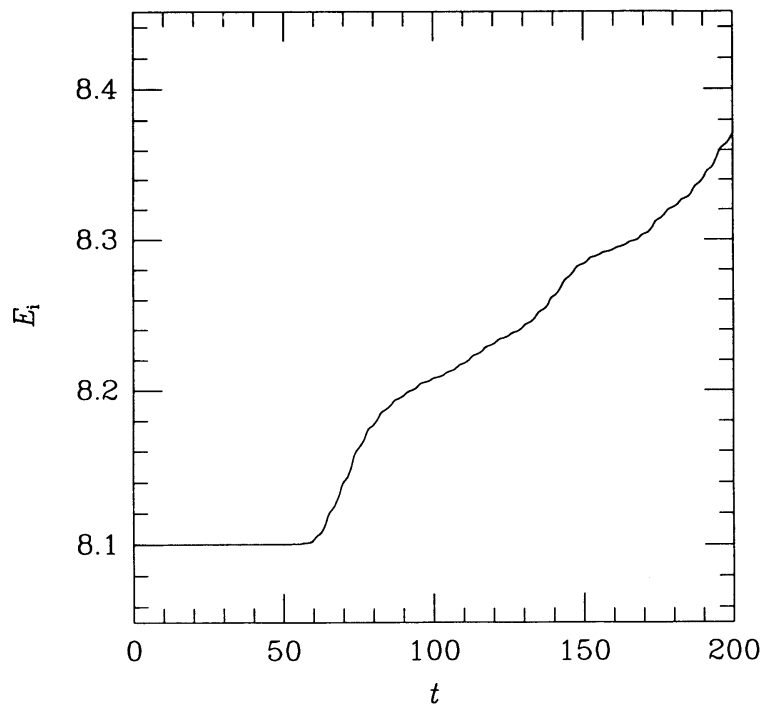

FIG. 6
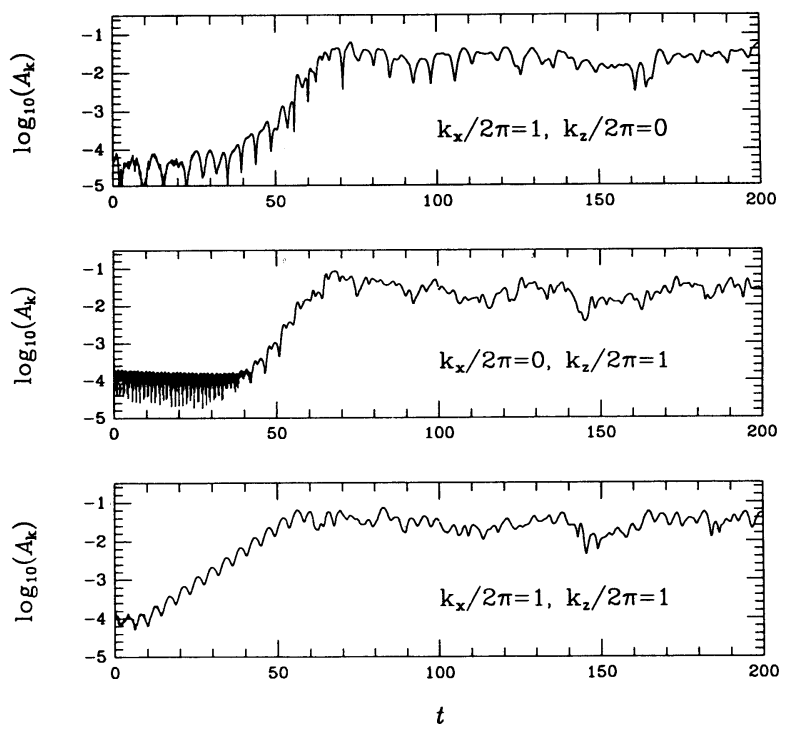

FIG. 7

Fig. 6.-Time evolution of the internal energy in run 2

FIG. 7.-Total Fourier amplitude of the velocity (eq. [65]) vs. time in run 2 for the three smallest nonzero wavenumbers 
that most of the turbulent energy resides at the largest scales. The prediction in equation (62) can be tested by these simulations because the energy irreversibly lost to the primary mode is ultimately dissipated and therefore turns up in the internal energy of the fluid. Figure 6 shows the evolution of the internal energy for run 2. From the plot, we estimate the energy absorption rate in the nonlinear regime to be $\dot{E}_{\text {int }} \sim 1.7 \times 10^{-3}$ in our dimensionless units. This is in reasonable agreement with the rate $2.6 \times 10^{-3}$ predicted by equation (62) if $f_{2}=1$. Analogous estimates for all eight runs are summarized in column (7) of Table 2 . From these numbers, we estimate $f_{2} \approx 0.6$.

In order to quantify the isotropy and spatial power spectrum of the saturated velocity field, we have estimated the Fourier amplitudes of the velocity components as

$$
A_{j}(\boldsymbol{k})=\left|\frac{1}{L^{2}} \int H_{x} H_{z} v_{j} \exp \left(i k_{x} x+i k_{z} z\right) d x d z\right|,
$$

where $j \in\{x, y, z\}$. Here $H_{x}$ and $H_{z}$ are the Hanning window functions (cf. Press et al. 1986)

$$
H_{x}=\frac{1}{2}\left[1-\cos \left(2 \pi \frac{x}{L}\right)\right], \quad H_{z}=\frac{1}{2}\left[1-\cos \left(2 \pi \frac{z}{L}\right)\right] .
$$

Windowing in $z$ was used because the flow is not periodic in that direction; it then seemed desirable to window in $x$, too, in order avoid artificial anisotropies in Fourier space. (However, windowing might have been avoided altogether if we had used sine and cosine transforms in $z$ rather than the usual. Discrete Fourier Transform.) We define the total Fourier amplitude

$$
A_{\boldsymbol{k}} \equiv\left[A_{x}^{2}(\boldsymbol{k})+A_{y}^{2}(\boldsymbol{k})+A_{z}^{2}(\boldsymbol{k})\right]^{1 / 2} .
$$

The energy per unit Fourier-space area $\left(\equiv d k_{x} d k_{z}\right)$ is $L^{2}\left|A_{\boldsymbol{k}}\right|^{2}$.

Figure 7 shows, for run $2, A_{\boldsymbol{k}}$ plotted against time at the three smallest nonzero wavenumbers, $(2 \pi / L, 0),(0,2 \pi / L)$, and $(2 \pi / L, 2 \pi / L)$. In the linear regime, $t \lesssim 60 / \Omega$, the mode at $\left(\left|k_{x}\right|,\left|k_{z}\right|\right)=( \pm 2 \pi / L, \pm 2 \pi / L)$ has the largest power, which confirms the visual impression given by the top gray-scale images in Figure 5 . In the nonlinear regime, the three modes have comparable power.

In Figure 8 we plot contours of $A_{k}$ averaged over the nonlinear phase of run 2. (The Fourier amplitudes were sampled at intervals $\Delta t=10$ during the period $100 \leq t \leq 200$ and then averaged.) The power appears remarkably isotropic in the $\left(k_{x}, k_{z}\right)$-plane, even though the source terms (eq. [35]) are quite anisotropic in $x$ and $z$.

In Figure 9 the time-averaged $A_{\boldsymbol{k}}$ is plotted against $k$ for runs 1 and 2, which differ in spatial resolution only, and for a similar simulation with $32 \times 32$ cells. Three cuts in the $\left(k_{x}, k_{z}\right)$-plane-along the lines $k_{z}=0, k_{z}=0$, and $k_{x}=k_{z}$-are shown. According to the conservative estimate of $\S 2.2$, our code can follow the development of turbulent structures occupying more than $\sim 10$ cells in each spatial direction. This suggests that the Fourier amplitude of the modes with $k \lesssim 3(2 \pi / L)$ in the simulations with $32 \times 32$ cells, $k \lesssim 6(2 \pi / L)$ with $64 \times 64$ cells, and $k \lesssim 12(2 \pi / L)$ with $128 \times 128$ cells is reproduced correctly. The specta in Figure 9 are indeed in good agreement below these wavenumbers.
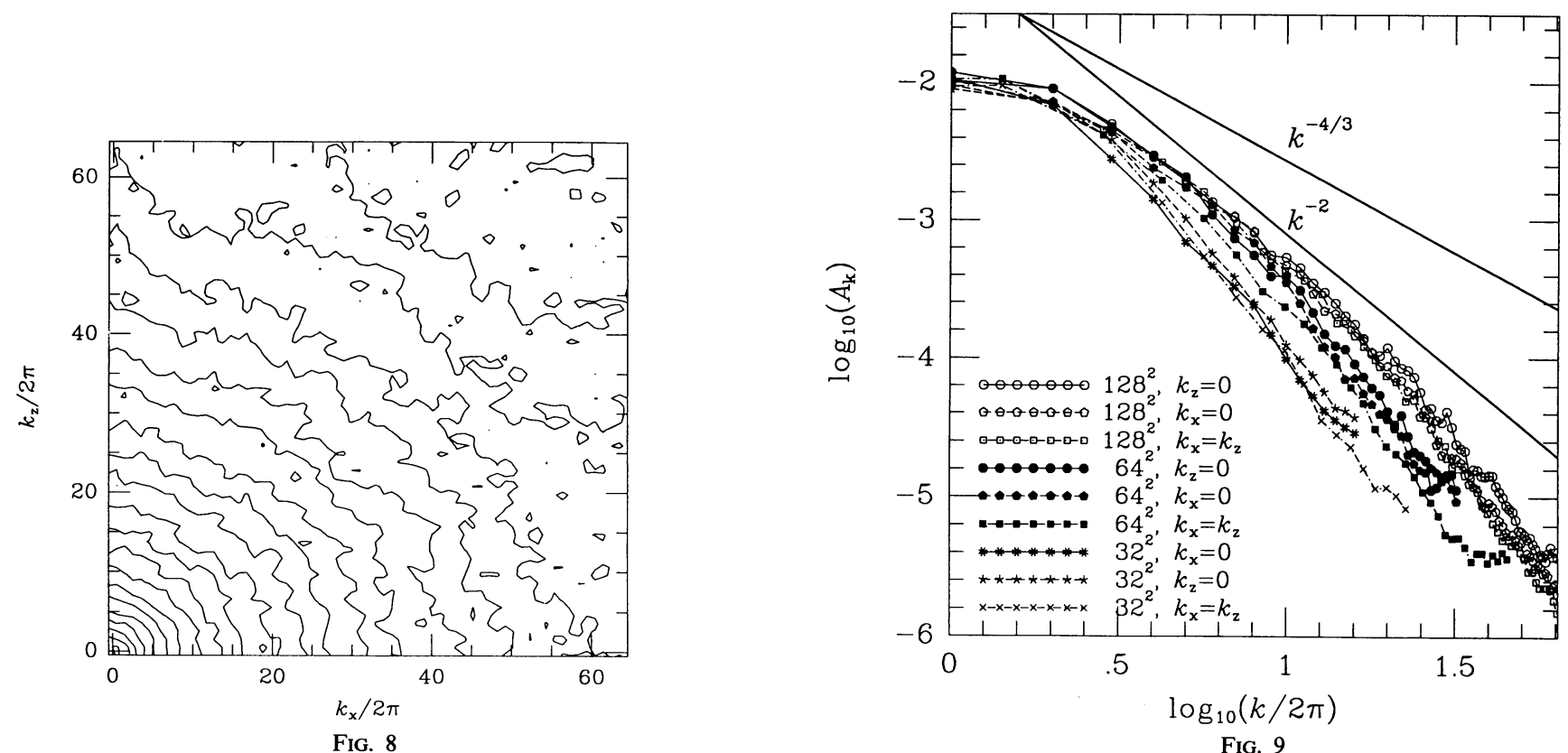

FIG. 8.-Contours of the Fourier-space velocity amplitude in the saturated phase of run 2

Fig. 9.-Fourier-space velocity amplitude vs. wavenumber in simulations with $64 \times 64$ cells (run 1), $128 \times 128$ (run 2 ), and $32 \times 32$. For each run, three cuts through Fourier space are shown: $k_{x}=0, k_{z}=0$, and $k_{x}=k_{z}$. For comparison, solid lines show $k^{-2}$ and $k^{-4 / 3}$ power laws. 
No single power-law accurately fits the data of Figure 9. The data are plausibly consistent with a trend toward $A_{\mathbf{k}} \propto k^{-2}$ or $k^{-4 / 3}$ at infinite resolution. A $k^{-2}$ spectrum was observed in two-dimensional simulations of compressible convection by Porter $\&$ Woodward (1992), as expected for free or forced two-dimensional turbulence shortward of the energy-injection length scale (Lilly 1971 and references therein). This assumes that vorticity is conserved as a scalar quantity. Because of the coupling of $v_{y}$ to $v_{x}$ via the Coriolis term, however, the component of vorticity perpendicular to the $(x, z)$-plane is not conserved in our simulations. If vorticity is not conserved and the turbulence is strong (the latter is unclear in our case), we may expect the Kolmogorov scaling, which in two-dimensions is $A_{k} \propto k^{-4 / 3}$.

Figure 10 displays the mass and angular momentum fluxes averaged over the plane $x=0$ (that is, $r=r_{0}$ ) for two realizations of run 2 differing only in the random noise introduced in the initial conditions. These fluxes are shown as cumulative averages over time: the rightmost point on each plot corresponds to an average over the entire run. The periodic $x$ (radial) boundary condition permits these fluxes to be nonzero. The mass flux is very small: at late times in both realizations, $\left\langle F^{(m)}\right\rangle \lesssim 10^{-5} \rho \delta v$; we are inclined to regard this as consistent with zero. The angular momentum flux is also small, though not as small (in a dimensionless sense) as the mass flux. We regard this, too, as being consistent with zero-especially since $\left\langle F^{(j)}\right\rangle$ appears to have opposite signs at late times in the two realizations shown in Figures $10 b$ and $10 d$.

\section{DISCUSSION}

We have learned from the simulations reported in $\S 4$ that when the modes saturate (that is, when their amplitudes cease to grow exponentially), the fluid continues to absorb energy from the tidal distortion. It was not obvious that this should be the case. If an anharmonic oscillator with 1 degree of freedom, such as a pendulum, is destabilized by a weak parametric term with a fixed driving frequency, the amplitude grows until the nonlinearity of the pendulum causes its natural frequency to shift out of the unstable frequency range. The instability has then saturated, and the pendulum ceases to absorb energy from the driver. (Daring children on playground swings delay saturation by adjusting the driving frequency as the amplitude increases.) This has not happened in our simulations. Evidently, many degrees of freedom are active, and saturation occurs by the transfer of energy from the primary mode to shorter wavelength modes, from which the energy is ultimately dissipated by numerical viscosity.

With the results of $\S 4$ in hand, we can make a quantitative estimate of the energy dissipation rate in a real disk due to these instabilities; more interestingly, we can estimate the tidal torque on the disk.

Combining equations (62) and (61), we have

$$
\dot{E}=f_{1}^{2} f_{2} s_{\max }^{3} k^{-2} \text {. }
$$

The smallest permitted values of $k$ for which the resonance condition (60) can be satisfied are $\sim \pi / H$, since $k_{z}$ is quantized in units of approximately this size by the boundary conditions at the surface of the disk. In our simulations, the minimal values of $k$ were a few

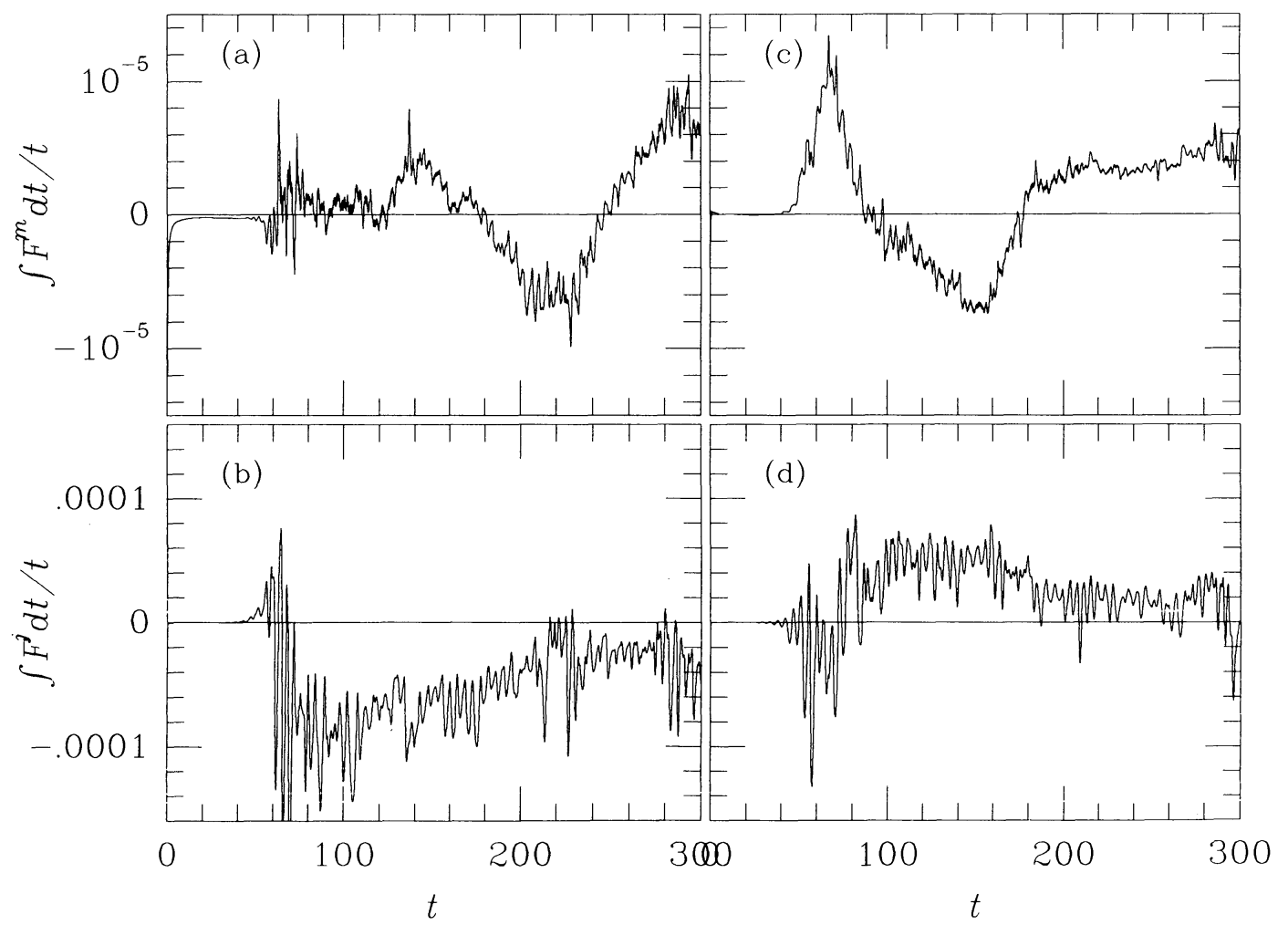

FIG. 10.-Cumulative time averages of the mass and angular momentum fluxes across the $x=0$ plane for two realizations of run 2 with different but identically distributed random noise in the initial conditions. 
times larger because we used a periodicity length $(L)$ 3-5 times smaller than $H$, except in run 7 . Nevertheless, we shall assume that the scaling of equation (66) can be extended to $k=\pi / H$.

Precise values for $s_{\max }$ are given in graphical form in Paper I, but we use here the asymptotic small-r growth rate (eq. [6]) and correct it by a dimensionless factor $f_{3} \sim 1$. (At $r / a=0.4$ and $M_{2} \ll M_{1}$, for example, $f_{3} \approx 2$.) Therefore, the predicted dissipation rate per unit mass is

$$
\dot{E}=\left(\frac{15}{4}\right)^{3} \pi^{-2} f_{1}^{2} f_{2} f_{3}^{3} \mu^{3} \frac{\Omega_{b}^{6}}{\Omega^{3}} H^{2}
$$

Here $\mu \equiv M_{2} /\left(M_{1}+M_{2}\right)$.

The tidal torque on a viscous disk has been considered by Papaloizou \& Pringle (1977, hereafter PP). These authors have shown by explicit calculation that a dissipation rate $\dot{E}$ produced by viscosity acting on the tidal "bulge" implies a retarding torque

$$
\dot{J}=\frac{-\dot{E}}{\Omega-\Omega_{b}} .
$$

The approximations with which PP treat the disk are different from those we employ, but the general result in equation (68) is equally true here, because it follows from the following simple (though not quite rigorous) argument: The energy (actually the Jacobian integral) $E_{\text {disk }}$ of the disk is conserved because the tidal potential of the secondary is constant in the frame rotating at $\Omega_{b}$. This is true even though the disk radiates energy from the surface, provided that the photons so radiated are considered still to "belong" to the disk. Therefore, energy deposited in the form of perturbed velocities (and then dissipated as heat) must be balanced by a withdrawal of some other form of energy from the fluid. The only fund of energy available is the orbital energy, so there must be a retarding torque per unit mass $\dot{J}$ that satisfies

$$
\int\left(\Omega-\Omega_{b}\right) \dot{j} \rho d^{3} r=\dot{E}_{\text {tot }},
$$

since the left-hand side represents the rate at which the torque does work against the disk as measured in the frame rotating at $\Omega_{b}$. The global result of equation (69) does not guarantee that equation (68) holds locally at each point in the disk, which is one reason that our argument is not rigorous.

Therefore, the torque per unit mass due to saturated parametric instabilities is

$$
\begin{aligned}
\dot{J} & =\left(\frac{15}{4}\right)^{3} \pi^{-2} f_{1}^{2} f_{2} f_{3}^{3} \mu^{3} \frac{\Omega_{b}^{6}}{\Omega^{4}} H^{2} \\
& \approx-320 \mu^{3} \frac{\Omega_{b}^{6}}{\Omega^{4}} H^{2} .
\end{aligned}
$$

Here $\Omega_{b}$ has been neglected compared with $\Omega$ in applying equation (68) because

$$
\frac{\Omega_{b}}{\Omega} \approx(1-\mu)^{-1 / 2}\left(\frac{r}{a}\right)^{3 / 2} \ll 1 \quad \text { if } \quad r \ll a .
$$

To obtain the final expression in equation (70), we have inserted our estimated values for the dimensionless coefficients (see $\S 4$ and Table 2): $f_{1} \approx 10, f_{2} \approx 0.6$, and $f_{3} \approx 1$.

It may be helpful to compare our result in equation (70) with the tidal torque on a viscous disk, which has been calculated by PP:

$$
\dot{J}_{\mathrm{PP}}=-\frac{9}{8} \frac{\zeta}{\rho} \mu^{2} \frac{\Omega_{b}^{4}}{\Omega^{3}}
$$

where $\zeta$ is the coefficient of bulk viscosity. ${ }^{5}$ To facilitate the comparison, we have simplified PP's expression for the torque by neglecting all but the $m=2$ component of the tide (which is the dominant one) and by evaluating at small $r / a$, so that $\Omega_{b} \ll \Omega$.

Note that the torques in equations (70) and (72) scale as different powers of radius within the disk $\left(r^{6}\right.$ and $r^{9 / 2}$, respectively). The numerical coefficients imply that parametric instabilities could be the more important effect in the outer part of the disk:

$$
j>j_{\mathrm{PP}} \quad \text { if } \quad \frac{r}{a}>0.15\left(\frac{\zeta}{\rho \Omega H^{2}}\right)^{1 / 3}\left(\frac{M_{1}}{M_{2}}\right)^{1 / 3} f_{3}^{-1} .
$$

One expects $\zeta /\left(\rho \Omega H^{2}\right) \lesssim 1$ for the same reasons that the Shakura $\&$ Sunyaev (1973) parameter $\alpha \equiv \eta /\left(\rho \Omega H^{2}\right) \lesssim 1$.

We ought to check, however, that the perturbed velocities $\delta v$ remain subsonic at these relatively large radii, since the scalings in equations (61) and (62) could be substantially altered if shocks became important. Since the sound speed $c_{s} \approx \Omega H$, it follows from

\footnotetext{
${ }^{5}$ PP found that the tidal torques due to shear and bulk viscosity are numerically nearly equal for equal values of the corresponding viscosity coefficients. The

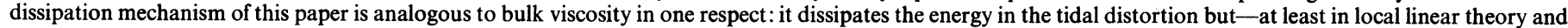
in these nonlinear two-dimensional simulations-does not transport angular momentum within the disk.
} 
equations (61) and (6) (with $k=\pi / H)$ that

$$
\frac{\delta v}{c_{s}} \approx \frac{15}{4 \pi} f_{1} f_{3}\left(\frac{\mu}{1-\mu}\right)\left(\frac{r}{a}\right)^{3}
$$

whence

$$
\frac{\delta v}{c_{s}}<1 \quad \text { if } \quad\left(\frac{r}{a}\right)<0.44\left(\frac{M_{1}}{M_{2}}\right)^{1 / 3} f_{3}^{-1 / 3} .
$$

Where they can be measured, disk outer radii are $\sim 0.4 a-0.6 a$ (Robinson 1976; Paczyński 1977; and references therein). We have reinserted the growth-rate factor $f_{3}$ in equations (73) and (74), because, although it is close to unity at small radii, it becomes abruptly larger as one approaches the streamline-crossing radius at the outer edge of the disk (see Paczyński 1977; Paper I). Equation (74) confirms that the parametric instabilities can dominate the tidal torque without necessarily forming shocks, and it may even suggest that these instabilities could play a role in determining the outer radius of the disk.

The upshot of this discussion is very encouraging. We have been able to calculate the tidal torque on an accretion disk in a binary system from first principles without appealing to an ad hoc viscosity, ${ }^{6}$ and our result is actually somewhat larger than the standard one for plausible values of the parameters.

Of course, our "first principles" may be oversimple. In particular, we have ignored the possibility of a relatively strong and partially ordered magnetic field in the disk, which could alter or even suppress the instabilities discussed here (see the discussion of this point in Paper I). Putting this concern aside, it remains true that the fundamental scalings in equations (61) and (62) have been fitted to numerical simulations rather than calculated from an analytic theory of the nonlinear turbulence (which may be within reach). The reader can see from Table 2 that there is some scatter in these scalings. Furthermore, three-dimensional simulations might have produced significantly different results. They might also have shown significant angular momentum transport, although at this point we hesitate to predict even the sign of the angular momentum flux. Three-dimensional simulations are now being attempted, and we hope to report on them in a future paper.

We thank Peter Goldrich, Hyesung Kang, and Scott Tremaine for helpful discussions. This work was supported by NASA's Astrophysics Theory Program under grant NAGW-2419, and by the David and Lucille Packard Foundation.

\section{APPENDIX A}

\section{EIGENVALUES AND EIGENVECTORS FOR THE HYDRODYNAMIC EQUATIONS}

Here we list the eigenvalues and the right and left eigenvectors of the Jacobian matrix $\boldsymbol{A}_{\boldsymbol{x}}(\boldsymbol{q})$, which are used to build the TVD scheme. The eigenvalues and eigenvectors of $\boldsymbol{A}_{y}(q)$ and $\boldsymbol{A}_{z}(q)$ are obtained by properly permuting the indices. The eigenvalues of $\boldsymbol{A}_{x}(\boldsymbol{q})$ are the characteristic velocities,

$$
\begin{aligned}
& a_{1}=v_{x}-c, \\
& a_{2}=v_{x}, \\
& a_{3}=v_{x}, \\
& a_{4}=v_{x}, \\
& a_{5}=v_{x}+c,
\end{aligned}
$$

where the sound speed is $c=(\gamma p / \rho)^{1 / 2}$.

The corresponding right eigenvectors are

$$
\begin{gathered}
\boldsymbol{R}_{1}=\left(\begin{array}{c}
1 \\
v_{x}-c \\
v_{y} \\
v_{z} \\
w-v_{x} c
\end{array}\right), \\
\boldsymbol{R}_{2}=\left(\begin{array}{c}
0 \\
0 \\
1 \\
0 \\
v_{y}
\end{array}\right),
\end{gathered}
$$

\footnotetext{
${ }^{6}$ The ability to exert a torque on the disk without an explicit viscosity is shared by other, previously discovered mechanisms: in particular, the spiral shocks reviewed by Matsuda et al. (1989) and Spruit (1989).
} 


$$
\begin{gathered}
\boldsymbol{R}_{3}=\left(\begin{array}{c}
1 \\
v_{x} \\
v_{y} \\
v_{z} \\
\theta / 2
\end{array}\right), \\
\boldsymbol{R}_{4}=\left(\begin{array}{c}
0 \\
0 \\
0 \\
1 \\
v_{z}
\end{array}\right), \\
1 \\
\boldsymbol{R}_{5}=\left(\begin{array}{c}
v_{x}+c \\
v_{y} \\
v_{z} \\
w+v_{x} c
\end{array}\right),
\end{gathered}
$$

where $w=(E+p) / \rho$ is the enthalpy and $\theta=v_{x}^{2}+v_{y}^{2}+v_{z}^{2}$.

The left eigenvectors which are orthonormal to the right eigenvector, $L_{l} \cdot \boldsymbol{R}_{m}=\delta_{l m}$, are

$$
\begin{aligned}
& \boldsymbol{L}_{1}=\left(\frac{(\gamma-1) \theta / 2+c v_{x}}{2 c^{2}},-\frac{(\gamma-1) v_{x}+c}{2 c^{2}},-\frac{(\gamma-1) v_{y}}{2 c^{2}},-\frac{(\gamma-1) v_{z}}{2 c^{2}}, \frac{(\gamma-1)}{2 c^{2}}\right), \\
& \boldsymbol{L}_{2}=\left(-v_{y}, 0,1,0,0\right), \\
& \boldsymbol{L}_{3}=\left(1-\frac{(\gamma-1) \theta}{2 c^{2}}, \frac{(\gamma-1) v_{x}}{c^{2}}, \frac{(\gamma-1) v_{y}}{c^{2}}, \frac{(\gamma-1) v_{z}}{c^{2}},-\frac{\gamma-1}{c^{2}}\right), \\
& \boldsymbol{L}_{4}=\left(-v_{z}, 0,0,1,0\right), \\
& \boldsymbol{L}_{5}=\left(\frac{(\gamma-1) \theta / 2-c v_{x}}{2 c^{2}},-\frac{(\gamma-1) v_{x}-c}{2 c^{2}},-\frac{(\gamma-1) v_{y}}{2 c^{2}},-\frac{(\gamma-1) v_{z}}{2 c^{2}}, \frac{(\gamma-1)}{2 c^{2}}\right) .
\end{aligned}
$$

In the TVD scheme, the flux is computed on grid-call boundaries, while the physical quantities are defined at cell centers. We use Roe's approximate Riemann solution (Roe 1981) to get averaged values of the physical quantities on the cell boundary:

$$
\begin{aligned}
v_{x, i+1 / 2} & =\frac{\sqrt{\rho_{i}} v_{x, i}+\sqrt{\rho_{i+1}} v_{x, i+1}}{\sqrt{\rho_{i}}+\sqrt{\rho_{i+1}}}, \\
v_{y, i+1 / 2} & =\frac{\sqrt{\rho_{i}} v_{y, i}+\sqrt{\rho_{i+1}} v_{y, i+1}}{\sqrt{\rho_{i}}+\sqrt{\rho_{i+1}}}, \\
v_{z, i+1 / 2} & =\frac{\sqrt{\rho_{i}} v_{z, i}+\sqrt{\rho_{i+1}} v_{z, i+1}}{\sqrt{\rho_{i}}+\sqrt{\rho_{i+1}}}, \\
w_{i+1 / 2} & =\frac{\sqrt{\rho_{i}} w_{i}+\sqrt{\rho_{i+1}} w_{i+1}}{\sqrt{\rho_{i}}+\sqrt{\rho_{i+1}}}, \\
c_{i+1 / 2}=\left\{( \gamma - 1 ) \left[w_{i+1 / 2}\right.\right. & \left.\left.-\frac{1}{2}\left(v_{x, i+1 / 2}^{2}+v_{y, i+1 / 2}^{2}+v_{z, i+1 / 2}^{2}\right)\right]\right\}^{1 / 2} .
\end{aligned}
$$

\section{APPENDIX B}

\section{PROCEDURE TO BUILD THE THREE-DIMENSIONAL TVD CODE}

The purpose of this appendix is to provide a short but complete description of the steps needed to build the three-dimensional TVD code from the eigenvalues and eigenvectors of Appendix A. For the details (that is, why and how each step works, etc.) consult the original reference (Harten 1983).

Aside from the fact that ours is a three-dimensional rather than a one-dimensional code, the procedure below is different in two places from the original one described by Harten (1983): First, the steepening operation for contact discontinuities has not been 
included (contact discontinuities are typically spread over two or three cells with steepening, six or seven cells without). No contact discontinuity forms in the simulations of this paper. Second, the numerical diffusion term for stabilizing expanding supersonic flows has not been included, since our flows are purely subsonic. With these two modifications, the speed of the code has been increased by $30 \%-40 \%$.

In updating the state vector $q^{n}$ to $q^{n+1}$, the fluxes along the $x, y$, and $z$ directions are computed and applied separately and successively as if the scheme were one-dimensional. The $x$-flux procedure is the following:

$$
\begin{gathered}
L_{x} \boldsymbol{q}_{i}^{n} \equiv \boldsymbol{q}_{i}^{n}-\frac{\Delta t}{\Delta x}\left(\bar{f}_{x, i+1 / 2}-\bar{f}_{x, i-1 / 2}\right), \\
\bar{f}_{x, i+1 / 2}=\frac{1}{2}\left[\boldsymbol{F}_{x}\left(\boldsymbol{q}_{i}^{n}\right)+\boldsymbol{F}_{x}\left(\boldsymbol{q}_{i+1}^{n}\right)\right]-\frac{\Delta x}{2 \Delta t} \sum_{k=1}^{5} \beta_{k, i+1 / 2} \boldsymbol{R}_{k, i+1 / 2}^{n}, \\
\beta_{k, i+1 / 2}=\left|\frac{\Delta t}{\Delta x} a_{k, i+1 / 2}^{n}+\gamma_{k, i+1 / 2}\right| \alpha_{k, i+1 / 2}-\left(g_{k, i}+g_{k, i+1}\right), \\
\gamma_{k, i+1 / 2}= \begin{cases}\left(g_{k, i+1}-g_{k, i}\right) / \alpha_{k, i+1 / 2} & \text { for } \\
0 & \alpha_{k, i+1 / 2} \neq 0, \\
g_{k, i}=\operatorname{sign}\left(\tilde{g}_{k, i+1 / 2}\right) \max \left\{0, \min \left[\left|\tilde{g}_{k, i+1 / 2}\right|, \tilde{g}_{k, i-1 / 2} \text { sign }\left(\tilde{g}_{k, i+1 / 2}\right)\right]\right\}, \\
\tilde{g}_{k, i+1 / 2}=\frac{1}{2}\left[\left|\frac{\Delta t}{\Delta x} a_{k, i+1 / 2}^{n}\right|-\left(\frac{\Delta t}{\Delta x} a_{k, i+1 / 2}^{n}\right)^{2}\right] \alpha_{k, i+1 / 2} .\end{cases}
\end{gathered}
$$

The time step $\Delta t$ is restricted by the usual Courant condition for stability, $\Delta t<\Delta x /\left(\left|v_{x, i+1 / 2}^{n}\right|+c_{i+1 / 2}^{n}\right)$. Analogous operators $L_{y}$ and $L_{z}$ are applied to take account of the $y$ and $z$ fluxes. Then the updated vector is

$$
q^{n+1}=L_{z} L_{y} L_{x} q^{n}
$$

In order to achieve second-order accuracy in time, the one-dimensional operators are permuted according to a Strange-type dimensional splitting (Strang 1968): $L_{z} L_{y} L_{x}, L_{x} L_{y} L_{z}, L_{x} L_{z} L_{y}, L_{y} L_{z} L_{x}, L_{y} L_{x} L_{z}$, and then $L_{z} L_{x} L_{y}$.

\section{APPENDIX C}

\section{PROCEDURE TO INCLUDE SOURCE TERMS IN THE TVD CODE}

Here we outline the procedure for including the source terms in equation (35) in the TVD code with second-order accuracy.

Step 0.-We have $\rho^{n}, v_{i}^{n}$, and $E^{n}$ at $t^{n}$.

Step 1.-We compute $\tilde{v}_{i}^{n}$ and $\widetilde{E}^{n}$ before calling the TVD subroutine:

$$
\begin{aligned}
\rho^{n} \tilde{v}_{i}^{n} & =\rho^{n} v_{i}^{n}+\frac{\Delta t}{2} S_{i}\left(\rho^{n}, v_{i}^{n}, E^{n}, t^{n}\right), \\
\tilde{E}^{n} & =E^{n}+\frac{\Delta t}{2} S_{E}\left(\rho^{n}, v_{i}^{n}, E^{n}, t^{n}\right) .
\end{aligned}
$$

Step 2.-We apply the TVD algorithm, ignoring the source terms, to obtain

$$
\begin{aligned}
\rho^{n+1} & =\rho^{n}+\Delta \rho^{\mathrm{TVD}}\left(\rho^{n}, \tilde{v}_{i}^{n}, \tilde{E}^{n}\right), \\
\left(\rho v_{i}\right)_{\mathrm{TVD}}^{n+1} & =\left(\rho^{n} v_{i}^{n}\right)+\Delta\left(\rho v_{i}\right)^{\mathrm{TVD}}\left(\rho^{n}, \tilde{v}_{i}^{n}, \tilde{E}^{n}\right), \\
E_{\mathrm{TVD}}^{n+1} & =E^{n}+\Delta E^{\mathrm{TVD}}\left(\rho^{n}, \tilde{v}_{i}^{n}, \tilde{E}^{n}\right) .
\end{aligned}
$$

Step 3.-We update the state vector using the source terms again:

$$
\begin{aligned}
\left(\rho v_{i}\right)^{n+1} & =\left(\rho v_{i}\right)_{\mathrm{TVD}}^{n+1}+\Delta t S_{i}\left(\tilde{\rho}^{n+1 / 2}, \tilde{v}_{i}^{n+1 / 2}, \tilde{E}^{n+1 / 2}, t^{n+1 / 2}\right) \\
E^{n+1} & =(E)_{\mathrm{TVD}}^{n+1}+\Delta t S_{E}\left(\tilde{\rho}^{n+1 / 2}, \tilde{v}_{i}^{n+1 / 2}, \tilde{E}^{n+1 / 2}, t^{n+1 / 2}\right)
\end{aligned}
$$


where

$$
\begin{aligned}
\tilde{\rho}^{n+1 / 2} & =\frac{\rho^{n}+\rho^{n+1}}{2}, \\
\tilde{v}_{i}^{n+1 / 2} & =\frac{v_{i}^{n}+v_{i}^{n+1}}{2}, \\
\tilde{E}^{n+1 / 2} & =\frac{E^{n}+E^{n+1}}{2}
\end{aligned}
$$

We now have $\rho^{n+1}, v_{i}^{n+1}$, and $E^{n+1}$ at $t^{n+1}$.

Note that in order to solve equations (C4)-(C8) previously, we would have to use an implicit scheme-or an iterative explicit scheme if the source terms were small enough to permit convergence. However, a single-step explicit scheme has been used instead because the source terms are comparatively small: we replaced $v_{i}^{n+1}$ and $E_{i}^{n+1}$ by $\left(v_{i}^{n+1}\right)_{\mathrm{TVD}}$ and $\left(E_{i}^{n+1}\right)_{\mathrm{TVD}}$ on the right-hand sides of equations (C7) and (C8).

\section{REFERENCES}

Bayly, B. J. 1986, Phys. Rev. Lett., 57, 2160

Craik, A. D. D., \& Criminale, W. O. 1986, Proc. R. Soc. Lond., A, 406, 13

Goodman, J. 1993, ApJ, 406, 596 (Paper I)

Harten, A. 1983, J. Comput. Phys., 49, 357

Kaisig, M. 1989a, A\&A, 218, 89 1989 b, A\&A, 218, 102

Landau, L. D., \& Lifshitz, E. M. 1967, Mechanics (3d ed.; Oxford: Pergamon)

Landman, M. J., \& Saffman, P. G. 1987, Phys. Fluids, 30, 2339

Lilly, D. K. 1971, J. Fluid Mech., 45, 395

Lubow, S. H., Pringle, J. E., \& Kerswell, R. R. 1993, ApJ, 419, in press (LPK)

Matsuda, T., Sekino, N., Shima, E., Sawada, K., \& Spruit, H. 1989, in The Theory of Accretion Disks, ed. F. Meyer et al. (Dordrecht: Kluwer), 355

Paczyński, B. 1977, ApJ, 216, 822

Papaloizou, J. C. B., \& Pringle, J. E. 1977, MNRAS, 181, 441 (PP)
Pierrehumbert, R. T. 1986, Phys. Rev. Lett., 57, 2157

Porter, D. H., \& Woodward, P. R. 1992, preprint

Press, W. H., Flannery, B. P., Teukolsky, S. A., \& Vetterling, W. T. 1986, Numerical Recipes: The Art of Scientific Computing (Cambridge: Cambridge Univ. Press)

Reynolds, O. 1883, Phil. Trans. R. Soc. Lond, A, 174, 935

Robinson, E. L. 1976, ARA\&A, 14, 119

Roe, P. L. 1981, J. Comput. Phys., 43, 357

Shakura, N. J., \& Sunyaev, R. A. 1973, A\&A, 24, 337

Spruit, H. C. 1989, in The Theory of Accretion Disks, ed. F. Meyer et al. (Dordrecht: Kluwer), 325

Strang, G. 1968, SIAM J. Num. Anal. 5, 506

Weinberg, S. 1972, Gravitation and Cosmology (New York: Wiley) 